
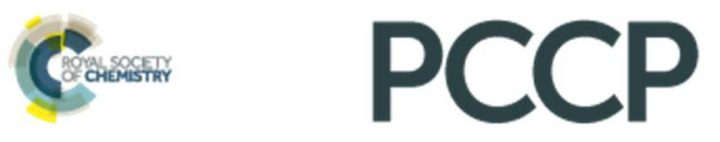

\title{
Recent Developments in Solid-State NMR Spectroscopy of Crystalline Microporous Materials
}

\begin{tabular}{|r|l|}
\hline Journal: & Physical Chemistry Chemical Physics \\
\hline Manuscript ID: & CP-PER-02-2014-000578.R1 \\
\hline Article Type: & Perspective \\
\hline Date Submitted by the Author: & n/a \\
\hline Complete List of Authors: & $\begin{array}{l}\text { Ashbrook, Sharon; University of St Andrews, School of Chemistry } \\
\text { Dawson, Daniel; University of St Andrews, School of Chemistry } \\
\text { Seymour, Valerie; University of St Andrews, School of Chemistry }\end{array}$ \\
\hline
\end{tabular}

\section{SCHOLARONE ${ }^{\text {TW }}$ \\ Manuscripts}




\section{'PCCP Perspective' Guidelines for Authors}

\section{PCCP}

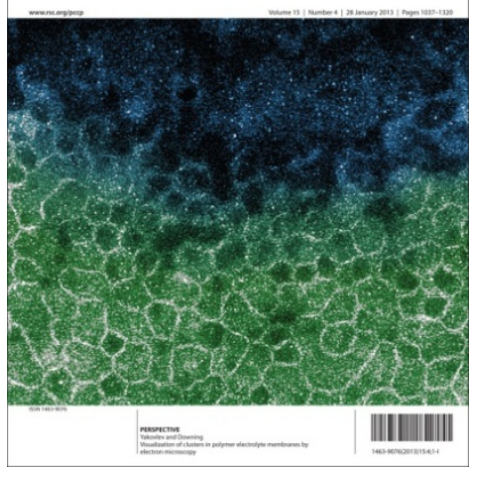

A 'PCCP Perspective' is a high profile review-type article that presents an authoritative state-of-the-art account of the selected research field.

It should be of interest to a broad spectrum of researchers and should be both enticing and easily readable to the nonexpert.

All Perspectives undergo a rigorous and full peer review procedure.

Perspectives can also include some new unpublished research and may be a more personal account.

Published 'PCCP Perspectives' are highly promoted.

A 'PCCP Perspective' must:

- Present a balanced account of the research field.

- Offer new insights into an area, through the presentation of new data, techniques, or a critical assessment of existing information.

- Close with clear conclusions and suggestions for future avenues of enquiry, including its impact on other fields - cross-fertilization of ideas is very important.

Additional guidelines:

- Length: 5000-10000 words, together with figures and tables. Flexibility is offered if justified. Shorter Perspective articles of less than 5000 words (3-4 journal pages) highlighting a topical area or important new development in an established field are also welcome. Authors can also make use of the Electronic Supplementary Information service.

- A brief biography and photo of the author(s) will appear with the published article.

- The author(s) can provide cover artwork for consideration. If chosen, a contribution to the additional production costs may be requested.

- $\quad$ Authors can also send a video abstract introducing the topic and themselves.

- Copyright: If the author(s) need to reproduce illustrations, tables or quotations from another source, it is their responsibility to obtain written permissions* from the copyright owner (normally the journal publisher).

- $\quad$ PCCP will not publish material which actively promotes the interest of a process, instrument, software or other intellectual property of actual or potential commercial value.

*Permission request forms available at: www.rsc.org/AboutUs/Copyright/Permissionrequests.asp. RSC Author Guidelines (including Ethical Guidelines): Uwww.rsc.org/publishing/journals/guidelines/ 


\section{Recent Developments in Solid-State NMR Spectroscopy of Crystalline Microporous Materials}

Sharon E. Ashbrook, Daniel M. Dawson and Valerie R. Seymour

School of Chemistry, EaStCHEM and St Andrews Centre for Magnetic Resonance, St Andrews KY16 9ST, United Kingdom

Email:sema@st-andrews.ac.uk

Invited Perspectives article for Phys. Chem. Chem. Phys. 


\begin{abstract}
Microporous materials, having pores and channels on the same size scale as small to medium molecules, have found many important applications in current technologies, including catalysis, gas separation and drug storage and delivery. Many of their properties and functions are related to their detailed local structure, such as the type and distribution of active sites within the pores, and the specific structures of these active sites. Solid-state NMR spectroscopy has a strong track record of providing the requisite detailed atomic-level insight into the structures of microporous materials, in addition to being able to probe dynamic processes occurring on timescales spanning many orders of magnitude (i.e., from $\mathrm{s}$ to $\mathrm{ps}$ ). In this Perspective, we provide a brief review of some of the basic experimental approaches used in solid-state NMR spectroscopy of microporous materials, and then discuss some more recent advances in this field, particularly those applied to the study of crystalline materials such as zeolites and metal-organic frameworks. These advances include improved software for aiding spectral interpretation, the development of the NMR-crystallography approach to structure determination, new routes for the synthesis of isotopically-labelled materials, methods for the characterisation of host-guest interactions, and methodologies suitable for observing NMR spectra of paramagnetic microporous materials. Finally, we discuss possible future directions, which we believe will have the greatest impact on the field over the coming years.
\end{abstract}




\section{Introduction}

Microporous materials, containing molecule-sized (typically $<2 \mathrm{~nm}$ ) channels and pores, have applications involving manipulation of adsorbed "guest" molecules, including storage, controlled release and chemical transformation (e.g., catalysis). ${ }^{1}$ In addition, their physical properties, such as expansion behaviour, luminescence and magnetism, may also be of interest. Microporous materials may be entirely organic (e.g., van der Waals crystals $^{2}$ ), carbonaceous (e.g., activated carbons), a mixture of organic and inorganic components (e.g., metal-organic frameworks, or $\mathrm{MOFs}^{3}$ ), or wholly inorganic (e.g., zeolites $\left.^{1}\right)$. This diversity makes it difficult to provide even a brief overview of the entire field and this article will focus just on MOFs and zeolite-related materials (zeotypes) and in particular the recent advances in their study using nuclear magnetic resonance (NMR) spectroscopy. Readers are referred to more comprehensive recent reviews for more detail. 10

The structures of microporous materials can generally be considered as nets, with cationic vertices and anionic links between the vertices, as shown schematically in Figure 1(a) for the sodalite (SOD) net. ${ }^{11}$ The nature of the vertices and links depends on the class of material in question, and the same underlying topology (i.e., network connectivity) can be constructed from many chemically-distinct species. Aluminosilicate zeolites are composed of $\mathrm{SiO}_{4}$ and $\mathrm{AlO}_{4}$ tetrahedra, connected by Si-O-Si and Si-O-Al linkages, as shown in Figure $1(\mathrm{~b}){ }^{12}$ (Al-O-Al linkages are generally disfavoured according to Lowenstein's rule, and typically absent at low Al content ${ }^{1}$ ), giving structural motifs such as chains, rings and cages, known as secondary building units (SBUs). The connectivity of the SBUs determines the pore size and structure of the zeolite. Zeolitic frameworks are classified by the International Zeolite Association (IZA), which currently recognises 218 framework types, although more are added every year. ${ }^{11}$ Aluminosilicate zeolites are formed by substituting $\mathrm{Al}^{3+}$ for $\mathrm{Si}^{4+}$ in the parent $\mathrm{SiO}_{2}$ framework, resulting in a negative framework charge, which is balanced by cations within the pores or Brønsted acid sites 
$\left(\mathrm{H}^{+}\right)$on the framework. Lewis acidity and/or redox behaviour, which can be important for catalysis, may be introduced by the substitution of different elements into the tetrahedral framework sites. ${ }^{1,7,8}$ Zeolites are typically prepared hydrothermally, with the reagents (Si and $\mathrm{Al}$ precursors, and a cationic species (e.g., group 1 or 2 metal ions, $\mathrm{NH}_{4}^{+}, \mathrm{HNR}_{3}^{+}$or $\mathrm{R}_{4} \mathrm{~N}^{+}$)) combined in an aqueous medium, and heated until the zeolite precipitates. The cations are often known as structure-directing agents (SDAs), as changing the cation can alter the topology of the zeolite. Organic solvents (solvothermal synthesis), ${ }^{15}$ ionic liquids (ionothermal synthesis) ${ }^{16}$ and solvent-free syntheses ${ }^{17}$ allow different reagents and reaction conditions to be used, potentially yielding different framework types. The precise size and geometry of the framework determines the available void space and surface area of the zeolite, both of which are crucial to the function of the material.

Aluminophosphate zeolites ${ }^{13}$ (AlPOs) have an overall neutral $\mathrm{AlPO}_{4}$ framework, as their component $\mathrm{AlO}_{4}$ and $\mathrm{PO}_{4}$ tetrahedra are in strict alternation, as shown in Figure 1(c), for AlPO-20. ${ }^{13}$ AlPOs may be synthesised hydrothermally, solvothermally and ionothermally, or using deep eutectic solvents (DESs). ${ }^{16,18}$ The (cationic) SDA typically remains in the pores of the AlPO after synthesis and its charge is balanced by the presence of $\mathrm{Al}$-bound $\mathrm{F}^{-}$or $\mathrm{OH}^{-}$, giving five- and/or six-coordinate $\mathrm{Al}$ species. "As-made" AlPOs may also contain water, which may be found in the pores or may attach to the framework Al. ${ }^{1}$ Calcination of an AlPO removes the SDA, anions and water, yielding the purely tetrahedral AlPO framework, as shown in Figure 1(c). ${ }^{1}$ AlPOs readily form many structures isomorphous to aluminosilicates, as well as some framework types that are (so far) unique to AlPOs. ${ }^{11}$ Chemical activity may be introduced to AlPOs by substitution of either isovalent or aliovalent cations for some of the Al or P. These materials are generally termed MeAPOs $(\mathrm{Me}=$ metal) or SAPOs (Si-substituted AlPOs) and have an activity comparable to zeolites for industrially-relevant processes. ${ }^{1}$ Alternatively, the active centres can be included in the pores of the framework or the "dangling" $\mathrm{P}-\mathrm{OH}$ and Al-OH groups of partially-condensed or "interrupted" frameworks. ${ }^{1}$ 
Gallophosphates (GaPOs) generally exhibit frameworks analogous to other zeolites, although some frameworks are unique to GaPOs. ${ }^{11}$ Like AlPOs, GaPOs are composed of strictly alternating $\mathrm{GaO}_{4}$ and $\mathrm{PO}_{4}$ tetrahedra, giving an overall neutral $\mathrm{GaPO}_{4}$ framework. Their synthesis is typically hydrothermal or solvothermal, with relatively few ionothermal syntheses reported. ${ }^{19}$ While GaPOs are structurally similar to AlPOs, calcined GaPOs can be more challenging to prepare, and many are thought to "collapse" when the SDA is removed. ${ }^{20,21}$ While the applications of GaPOs are less well explored, some GaPOs, particularly the large-pore cloverite, have been demonstrated to be catalytically active. ${ }^{22}$

MOFs contain inorganic SBUs connected by polytopic (multiply-connecting) organic "linkers". ${ }^{1,3,10}$ For example, the SOD-type ZIF-8 contains $\mathrm{Zn}^{2+}$ ions connected by imidazolate anions, as shown in Figure $1(\mathrm{~d}){ }^{11,14}$ MOFs are typically synthesised hydrothermally or solvothermally, although DESs have also been used. ${ }^{16}$ The SBUs may be mononuclear, or larger clusters or chains of metal ions. The great structural diversity of MOFs stems from the vast number of possible combinations of SBUs and linkers. The modular nature of MOFs (i.e., the SBUs and linkers may be considered as separate entities) allows the synthesis of series of isoreticular (possessing the same underlying connectivity) structures, ${ }^{1,3}$ with pore volumes and chemistry that may be "tuned" to the desired guest. The required chemical functionality may be introduced by three routes; direct synthesis, post-synthetic modification, or in situ modification, as shown in Figure 1(e). ${ }^{1,3}$ In many cases, direct synthesis is not possible, either because of undesired side reactions of the desired functionality or because the steric bulk of the functional group hinders framework formation. In such cases, post-synthetic modification of the framework may be achieved by installing a precursor functional group during synthesis and then transforming this once the framework has formed. ${ }^{23}$ In situ linker modification, through desired side reactions of the linker, provides a further avenue to the functionalisation of MOFs. ${ }^{24}$ The SBUs of many MOFs contain metal-bound solvent molecules, which may be removed by heat/vacuum treatment to generate "coordinatively unsaturated" sites (CUSs). CUSs are typically reactive, and will bind any suitable ligands. The CUSs, therefore, represent an 
additional target for modification, either by using a different metal in the SBU or using mixed-metal SBUs. ${ }^{1,3}$ In recent years, MOFs have found applications including medicine, gas separation/purification and storage, catalysis, polymerisation and fuel cells. ${ }^{25}$ In addition, physical properties such as magnetism, luminescence and extreme expansion mean that MOFs may have applications including magnetic resonance imaging (MRI), data storage, sensing and communication technology and construction. ${ }^{25}$

The structural characterisation of microporous materials involves many techniques, particularly crystallographic diffraction and solid-state NMR spectroscopy. Solid-state NMR spectroscopy is ideally suited to the study of microporous materials, as most contain multiple NMR-active nuclei, as summarised in Table 1. A combination of Bragg diffraction and solid-state NMR spectroscopy is particularly useful, since Bragg diffraction techniques can determine the long-range "average" periodic structure, whereas solid-state NMR can provide detailed, element-specific, information on the local structure, disorder and motion present. Combining these two complementary techniques, in an approach often termed "NMR crystallography", can lead to a much deeper understanding of the structure of the material than either technique alone could provide. ${ }^{26}$ In support of these experimental methods, quantum chemical calculations can provide insight into structural features that are difficult to understand by experimental methods alone. ${ }^{9,27,28}$

In this Perspective we will first introduce the basic experimental approaches to studying microporous materials using NMR spectroscopy. We will then discuss some of the recent synthetic, methodological, theoretical and technological advances in this field, before considering the possible directions of future research efforts. We will restrict our discussion to the investigation of MOFs and zeotypes only.

\section{Basic Approaches to NMR of Microporous Materials}

Unlike in solution, NMR spectra of solids are affected by anisotropic (orientation- 
dependent) interactions, including dipolar couplings and chemical shift anisotropy (CSA), which broaden the spectral lines, and hinder the extraction of information. ${ }^{29}$ A range of methods, including magic-angle spinning (MAS), decoupling and cross polarisation (CP), are commonly used to improve spectral resolution and/or sensitivity. ${ }^{29}$ Once highresolution spectra have been obtained, the sensitivity of the isotropic chemical shift (usually termed $\delta_{\text {iso }}$ ) to the atomic-scale environment is able to provide a range of simple structural information for microporous solids. For spin $\mathrm{I}=1 / 2$ nuclei, such as ${ }^{29} \mathrm{Si}$ in zeolites, ${ }^{31} \mathrm{P}$ in phosphates and ${ }^{13} \mathrm{C}$ in MOFs, the number of distinct resonances provides information on the symmetry of the structure, thereby supporting (or disproving) possible space group suggestions. In addition, ${ }^{13} \mathrm{C}$ NMR spectra are also able to confirm the type and relative ratios of organic linkers present in MOFs, and the nature of any organic guest species within the pores of each type of microporous material. This can be seen in Figure 2(a), where ${ }^{13} \mathrm{C}$ MAS NMR spectra of two $\mathrm{Sc}_{2} \mathrm{BDC}_{3}$ MOFs (where BDC = benzene-1,4dicarboxylate), with unfunctionalised and functionalised linkers, respectively, are shown. ${ }^{30}$ For the unfunctionalised MOF, it is clear from the carboxylate region ( 170-180 ppm) that there are two distinct dicarboxylate linkers (ratio $1: 2$ ). Although the spectra for the functionalised (amino-substituted) MOFs are more complicated, the peak at 151 ppm shows that the functionalised linker has been incorporated intact into the MOF. In general, the differences observed in chemical shift in microporous materials result from changes in the local geometry; for example, it has been shown that the ${ }^{29} \mathrm{Si}$ isotropic chemical shift in zeolites is sensitive to both changes in the T-O-T angle and the Si-O bond distances, and to changes in the next nearest neighbour (NNN) environment. ${ }^{1,45}$ For aluminosilicate zeolites, the 5-6 ppm change in chemical shift observed with each Al NNN has long been exploited to determine $\mathrm{Si} / \mathrm{Al}$ ratios and ordering - where it is usually assumed that Lowenstein's rule (i.e., the absence of Al-O-Al linkages described above) holds. ${ }^{1,45}$ Perhaps less well known is the observation of small, but significant, downfield shifts in ${ }^{31} \mathrm{P}$ NMR spectra of phosphate frameworks with a change in the coordination environment of the Al NNN species. $^{31}$ 
For quadrupolar nuclei, i.e., those with $\mathrm{I}>1 / 2$, such as ${ }^{27} \mathrm{Al},{ }^{17} \mathrm{O}$ and ${ }^{71} \mathrm{Ga}$, MAS does not result in a high-resolution spectrum, as it cannot remove completely the second-order quadrupolar broadening (which has a more complex angular dependence than the dipolar or CSA interactions). ${ }^{29,32}$ Despite the line broadening observed, if the magnitude of the quadrupolar coupling, $\mathrm{C}_{\mathrm{Q}}$, remains reasonable, valuable information can still be obtained. NMR spectroscopy of quadrupolar nuclei is useful for studying zeotype framework cations, metal cations within zeolite pores and the metal centres in MOF frameworks. For example, significant differences in chemical shift are observed for ${ }^{27} \mathrm{Al}$ and ${ }^{71} \mathrm{Ga}$ in zeolites and/or phosphate frameworks when the coordination number is changed. This can be seen in Figure 2(b), where the ${ }^{27} \mathrm{Al}$ spectrum of as-made JDF-2 shows four- and fivecoordinate $\mathrm{Al}$, while only four-coordinate $\mathrm{Al}$ is present in the calcined form (AlPO-53(B)). ${ }^{33}$ It has also been shown that ${ }^{45} \mathrm{Sc} N \mathrm{NMR}$ is a good probe of the local coordination environment in Sc-based MOFs, ${ }^{30,34,35}$ with sharp lines (i.e., small quadrupolar coupling) observed for $\mathrm{Sc}_{2} \mathrm{BDC}_{3}$, which contains isolated $\mathrm{ScO}_{6}$ units, but broader lines observed for MIL-53(Sc), which contains chains of $\mathrm{ScO}_{6}$ octahedra. However, for MOFs containing trimeric $\mathrm{Sc}_{3}\left(\mu_{3} \mathrm{O}\right)$ units (e.g., MIL-88(Sc) and MIL-100(Sc)) the effects of structural disorder (of the two waters and hydroxyl attached to the trimer unit) are easily apparent, with broadened and asymmetric lineshapes observed. ${ }^{35}$ For many AlPOs and GaPOs, the ratio of the intensities of the resonances for four-, five- and six-coordinate $\mathrm{Al} / \mathrm{Ga}$ is able to provide information on the position of the charge-balancing anions (e.g., $\mathrm{OH}^{-}$or $\mathrm{F}^{-}$) attached to the framework, i.e., whether they are terminal or bridging in nature.

It is possible to remove the quadrupolar interaction completely, but more complex approaches are required than sample rotation about a single angle. Double rotation (DOR) experiments, $^{36}$ where the sample is rotated about two angles simultaneously, have been utilised for ${ }^{27} \mathrm{Al}$ and ${ }^{17} \mathrm{O}$ in both zeolites and phosphates, and for ${ }^{71} \mathrm{Ga}$ in ULM-18. ${ }^{37-42}$ The simplicity of DOR (where the high-resolution spectrum is obtained in real time) is appealing, but the applicability of the technique has been limited by the slow spinning speed of the outer (larger) rotor, and the need for a specialist probehead. A more popular 
approach, using only a conventional probehead, is the multiple-quantum (MQ) MAS experiment, $^{43}$ where the quadrupolar broadening is removed by a combination of MAS and the correlation of multiple-quantum (usually triple-quantum, i.e., $m_{I}=+3 / 2 \leftrightarrow m_{I}=-$ $3 / 2$ ) and single-quantum transitions in a two-dimensional experiment. The result is a twodimensional spectrum containing a series of ridge lineshapes (one for each species), with an isotropic spectrum obtained from a projection onto the orthogonal axis. The resolution advantage of MQMAS is clear in Figure 2(c), where ${ }^{45}$ Sc NMR spectra of two forms of MIL53(Sc) are shown. ${ }^{34}$ Although the MAS spectra look fairly similar (each containing broad lineshapes, with features characteristic of quadrupolar broadening), the MQMAS spectra are quite different. For the dehydrated form the two Sc sites are clearly resolved, while for the hydrated form the change in symmetry is clear, with two resonances observed now with a $1: 3$ ratio. MQMAS has been widely applied to zeolites, phosphates and MOFs in recent years, enabling the number of distinct framework or cationic species to be identified, and phase transitions to be investigated..$^{4-8}$ The satellite-transition (ST) MAS experiment $^{4,45}$ offers a similar, but alternative, approach, with the correlation of two different single-quantum transitions (i.e., central $\left(\mathrm{m}_{\mathrm{I}}=+1 / 2 \leftrightarrow \mathrm{m}_{\mathrm{I}}=-1 / 2\right)$ and satellite $\left(\mathrm{m}_{\mathrm{I}}\right.$ $=-3 / 2 \leftrightarrow \mathrm{m}_{\mathrm{I}}=-1 / 2$ and $\left.\mathrm{m}_{\mathrm{I}}=+1 / 2 \leftrightarrow \mathrm{m}_{\mathrm{I}}=+3 / 2\right)$ transitions. Although STMAS usually has inherently higher sensitivity than MQMAS, it is significantly more challenging to implement, requiring a very accurate setting (to $54.736^{\circ} \pm 0.002^{\circ}$ ) of the spinning axis, accurate pulse timings and very stable sample spinning. ${ }^{45}$ This has generally limited its application in comparison to MQMAS, although it has been applied to phosphate-based frameworks. ${ }^{4,5,46}$

Although useful structural information can be obtained from high-resolution spectra, more detailed information can be obtained by exploiting the interactions between nuclei, through experiments that "recouple" a particular interaction or that transfer magnetisation between nuclear species. ${ }^{29}$ Experiments that use the dipolar coupling enable internuclear distances or proximities to be probed, while those that use the scalar coupling provide information on covalent connectivity. A popular choice is to reintroduce the 
dipolar coupling, in experiments such as rotational echo double resonance (REDOR) ${ }^{47}$ and transfer of populations in double resonance (TRAPDOR) ${ }^{48}$ allowing the measurement of internuclear distances between pairs of spins. These experiments have recently been applied to study framework-cation interactions in zeolites and cation-anion interactions in phosphates $^{4,549,50}$ as well as the ordering and distribution of functional groups within MOFs. ${ }^{51}$ An alternative approach involves recording a two-dimensional spectrum, where magnetisation is transferred from one spin to a second. These correlation experiments can be classified into two main types; homonuclear, where the two spins are of the same species, and heteronuclear, where the two spins are different nuclides. Common applications for microporous materials have included ${ }^{29} \mathrm{Si} /{ }^{29} \mathrm{Si}$ COSY and INADEQUATE experiments to probe through-bond connectivity in high-silica zeolites, ${ }^{4,5,7}$ and ${ }^{27} \mathrm{Al} /{ }^{31} \mathrm{P}$ heteronuclear correlation experiments (via both the dipolar (i.e., HETCOR) and J (i.e., INEPT-based) interactions) for aluminophosphates. ${ }^{4,5,52}$ Challenging applications include the recent use of ${ }^{27} \mathrm{Al} /{ }^{31} \mathrm{P}$ HETCOR correlation experiments (with magnetisation transfer via the dipolar coupling) to probe the connectivity in complex zeotypes, where disorder of the SDA or charge-balancing anions is present. ${ }^{4,53,54}$ The transfer of magnetisation was achieved simply using CP, but additional techniques were used to improve the sensitivity. More recently, ${ }^{27} \mathrm{Al} /{ }^{27} \mathrm{Al}$ correlation experiments have been utilised to study microporous materials $s^{4,5,55}$ - although the presence of the quadrupolar broadening in both dimensions of the spectra can hinder resolution, and recoupling interactions between two quadrupolar nuclei can be somewhat complicated. Furthermore, pulse sequences that combine MQMAS (or STMAS) with magnetisation transfer, ensuring that both dimensions of many heteronuclear correlation experiments have high resolution, have been introduced and have been applied to microporous materials, particularly AlPOs and, more recently, GaPOs. $^{4,5,56-58}$

In recent years the advent of higher magnetic field strengths has allowed a wider range of nuclei to be studied routinely, including those more challenging species with low gyromagnetic ratio, lower natural abundance or larger quadrupolar broadening. For 
example, high magnetic field (21.1 T) enabled the first ${ }^{67} \mathrm{Zn}$ studies of MOFs (specifically for zeolitic imidazolate frameworks, or ZIFs) to be performed recently. ${ }^{59}$ This work demonstrated that different sites could be differentiated from the MAS spectra, and that the ${ }^{67} \mathrm{Zn}$ NMR parameters were sensitive to the presence and nature of guest molecules within the cavities. High magnetic field (also $21.1 \mathrm{~T}$ ) was also used to study ${ }^{25} \mathrm{Mg}$ in the $\mathrm{Mg}$ form of the MOF, CPO-27 (sometimes referred to as MOF-74), ${ }^{60}$ where the removal of a water molecule activates the MOF, enabling a direct interaction between $\mathrm{Mg}$ and the guest species adsorbed. Zhu et al. have used both $9.4 \mathrm{~T}$ and $21.1 \mathrm{~T}$ to investigate ${ }^{91} \mathrm{Zr}(\mathrm{I}=5 / 2)$ NMR spectroscopy of microporous zirconium silicate frameworks (AM-2 and AV-3) and ${ }^{25} \mathrm{Mg}$ NMR of a magnesium-substituted AlPO framework (MgAPO-20). ${ }^{61}$ In the cases above spectra were acquired for static samples, owing to the significant quadrupolar broadening. Such "wideline" experiments have become more common over recent years, particularly at high field where the second-order quadrupolar broadening is reduced (as it is proportional to $1 / \mathrm{B}_{0}$ ). For very wide spectral lineshapes the limited excitation bandwidth of the radiofrequency pulses can be a problem. This has been overcome by acquiring the spectrum in a series of separate experiments with the transmitter frequency varied for each acquisition (sometimes referred to as "frequency-stepped acquisition" or "spin-echo mapping"), ${ }^{29}$ after which the separate spectra are co-added to produce the final spectrum. More recently, the use of adiabatic WURST ${ }^{62}$ pulses has been shown to improve the excitation bandwidth, and enable much broader lines to be acquired in a single spectrum. In many cases, the sensitivity of wideline spectra is improved by using Carr-PurcellMeiboom-Gill (CPMG) ${ }^{63,64}$ echo trains.

An additional hardware improvement in recent years has been the introduction of MAS probes capable of faster sample spinning rates. This is generally achieved by using smaller sample rotors; for example the fastest available spinning rates for commercial probes $(\sim 100 \mathrm{kHz})$ are obtained using rotors with a 1-mm outer diameter, which are able to hold $0.8 \mu \mathrm{l}$ of sample. Fast MAS is required for removing larger interactions, and is particularly useful for ${ }^{1} \mathrm{H}$ or ${ }^{19} \mathrm{~F}$ NMR, where significant dipolar interactions are observed 
between closely-spaced and strongly-coupled abundant spins. Using fast MAS it is possible to resolve different ${ }^{1} \mathrm{H}$ signals in microporous materials - for example, ${ }^{1} \mathrm{H}$ MAS at $62.5 \mathrm{kHz}$ was able to resolve at least 5 different ${ }^{1} \mathrm{H}$ species in UiO-66(Zr). ${ }^{6,65}$

\section{Recent Advances in Applications to Microporous Materials}

The combination of improvements in pulse sequence development, magnet technology and probe hardware have advanced the characterisation of microporous materials using solid-state NMR considerably over the last decade. While these improvements and the subsequent applications they have enabled are too numerous to discuss in detail, some of the main areas of recent interest are discussed below.

\subsection{First-Principles Calculations and NMR Crystallography}

The presence of anisotropic interactions in solid-state NMR spectra generally hinders the extraction of accurate structural information. Techniques to improve resolution, such as MAS or high-power decoupling, may also remove other information (e.g., the isotropic J coupling in the case of decoupling) that could aid structure solution. In an attempt to improve the quality and quantity of information that is easily available from NMR spectra, many experimentalists have started to exploit first-principles calculations, where NMR parameters can be predicted for a known structure or proposed structural model. Many of the approaches used are based on density functional theory (DFT), as this provides a cost-effective and efficient approach. Considerable interest was generated by the introduction of the Gauge-Including Projector Augmented Wave, or GIPAW, approach by Pickard and Mauri in 2001, ${ }^{66}$ as this enabled the calculation of accurate chemical shielding and quadrupolar interactions (using DFT) under periodic boundary conditions, an inherently intuitive approach for solid-state structures. This avoids the need, as in earlier methods, to approximate a solid as a cluster, centred on the atom of interest, with any "dangling" bonds terminated (typically by hydrogen), thereby perturbing the local 
structure. The initial GIPAW paper was cited 455 times between its publication in 2001 and the end of 2013, but notably many of these citations arise from the experimental NMR community, showing the wide impact of this work. GIPAW has been successfully applied to the calculation of NMR parameters for a wide variety of materials including organic materials, glasses, ceramics, minerals and polymers. ${ }^{9,27,28}$

For ordered solids, calculations can be used to help with spectral assignment and interpretation, and, in some cases, to aid experimental acquisition by prior prediction of the NMR parameters. Specifically for microporous materials, the calculation of ${ }^{27} \mathrm{Al}$ and ${ }^{31} \mathrm{P}$ NMR parameters was able to help assign the spectra of calcined and as-made AlPO-14, where distinct $\mathrm{Al}$ and $\mathrm{P}$ species have very similar local geometry, preventing the use of simple empirical relationships to provide a conclusive and unambiguous spectral assignment. ${ }^{67}$ Similarly, the 10 inequivalent oxygen sites in the ${ }^{17} \mathrm{O}$ spectrum of the $\mathrm{SiO}_{2}$ zeolite ferrierite were able to be assigned using GIPAW calculations. ${ }^{68}$ DFT calculations were also crucial in understanding the reversible phase transition that was observed in situ during NMR experiments on AlPO-53(A). These were able to show that this transition involved dehydration of the material to produce JDF-2. The two materials have the same framework topology and SDA (methylammonium), but differing amounts of water. ${ }^{33}$ Other examples include the calculation of ${ }^{1} \mathrm{H},{ }^{13} \mathrm{C},{ }^{17} \mathrm{O},{ }^{19} \mathrm{~F},{ }^{27} \mathrm{Al},{ }^{31} \mathrm{P},{ }^{71} \mathrm{Ga},{ }^{45} \mathrm{Sc},{ }^{67} \mathrm{Zn}$ and ${ }^{91} \mathrm{Zr}$ NMR parameters in zeotypes and MOFs. ${ }^{9,27,28}$

A pre-requisite for the calculation of NMR parameters is an initial structure or structural model. In many cases this is obtained from diffraction measurements, although some may be produced from prior computational work. The accuracy of diffractionderived structures depends upon the type of experimental measurement (i.e., single crystal or powder diffraction), whether experiments have been carried out on laboratory instruments, or using synchrotron or neutron sources, and whether the position of light atoms have been refined explicitly or simply added post refinement. This latter point is particularly important for as-made AlPOs and MOFs, where organic molecules are present 
in the structure. For example, for AlPO- $14,{ }^{67,69}$ poor agreement between experiment and calculation was obtained, unless the structural models were "optimised" (typically achieved by reducing the forces upon the atoms) prior to the calculation of the NMR parameters. Although the structural changes resulting from this optimisation were small, this did produce significant changes in the NMR parameters, reflecting the sensitivity of NMR as a probe of local structure. Similar optimisation was employed for a range of other zeotypic frameworks to obtain good agreement between experiment and calculation. ${ }^{9,31,70}$ It should be noted that for GIPAW calculations on AlPO- $15^{71}$ good agreement was obtained using the structural model from diffraction. However, in this case the structure was determined using synchrotron measurements on a single crystal, and almost no change was observed upon structural optimisation using DFT.

Any optimisation of structures or models using DFT must be undertaken with caution, and some thought given to the exact optimisation procedure used. It is possible to only optimise the position of light atoms (e.g., H), which can be difficult to locate accurately using X-ray diffraction methods. Alternatively, all atomic positions may be varied, as may the size and shape of the unit cell. However, many DFT calculations do not adequately describe long-range dispersion forces, leading to potential inaccuracies in the cell size if these are under represented. While possibly not a problem for dense, crystalline solids, this may be of greater importance for the more flexible microporous frameworks, particularly for MOFs and zeotypes with organic molecules within the pores, where dispersion interactions may have a significant effect upon the structure. It has been shown that optimisation of a Zn-based MOF using basic DFT calculations resulted in a cell expansion of $\sim 12 \%{ }^{72}$ while an expansion of $\sim 4 \%$ was obtained for MIL-53(Al)..$^{73}$ Although it is possible to constrain the cell parameters, this will not be possible when considering any sort of substitutions into, or variations of, a structure. Recent approaches to include dispersion interactions in DFT calculations have been introduced; the use of semiempirical dispersion correction (SEDC) schemes (or DFT-D) ${ }^{74}$ was shown to produce cell sizes in much better agreement with experiment for the two MOFs described above.72,73 
These schemes have also been shown to be applicable to AlPO-based frameworks, although the differences between the structures obtained when different optimisation methods are used are smaller than those seen for the more flexible MOFs. ${ }^{70}$

Although calculations can aid spectral assignment for ordered crystalline solids, in these cases their main role is often one of supporting or confirming experimental measurements. However, the real power of such calculations lies in their ability to provide structural insight for materials with less well-characterised structures, where potential models can be evaluated by considering the agreement between predicted NMR parameters and those observed experimentally. Although it is usually the combination of diffraction and NMR spectroscopy that is often referred to as NMR crystallography, as described previously, in many cases additional insight can be gained by using firstprinciples calculations to bridge between the two techniques. (This has also been referred to as Structure elucidation by coMbining mAgnetic Resonance, compuTation modEling and diffRaction, or "SMARTER" Crystallography). ${ }^{75}$ Although the framework topology for many microporous materials can be determined using diffraction, the framework cation disorder, the position of adsorbed molecules and/or water and the position of chargebalancing anions attached to the framework is considerably more challenging to establish. This combined approach has been applied to study phosphates, zeolites and MOFs, supporting or disproving possible structural models. ${ }^{9,31,76}$ One recent example of an NMR crystallographic approach for microporous materials is the investigation of the position of the charge-balancing hydroxyl groups in the as-made aluminophosphate framework, STA-2. ${ }^{31}$ It was possible to determine that the two hydroxyls per unit cell are bridging between $\mathrm{Al}$ species, from the relative ratio of four- and five-coordinate $\mathrm{Al}$ in the ${ }^{27} \mathrm{Al} \mathrm{MAS}$ spectrum (shown in Figure 3(a)), and MQMAS experiments showed that the hydroxyls bridged between Al1 and Al2 species. Diffraction measurements showed additional electron density within the "cancrinite" cages of the structure, suggesting that the hydroxyls could be found on two of six symmetry-related sites. By calculating NMR parameters for possible structural models and comparing them to an experimental $\mathrm{Al} / \mathrm{P}$ 
two-dimensional correlation spectrum (Figure 3(b)), it was possible to show that only one hydroxyl was found per cage and that not all hydroxyl arrangements were equally likely, but certain arrangements were favoured. ${ }^{31}$ Other, extremely elegant examples of the NMR crystallographic approach were demonstrated recently by Taulelle and co-workers for AlPO-cloverite and AlPO-CJ2. ${ }^{77,78}$ In each case, the framework topology was determined primarily using powder diffraction methods (supported by ${ }^{27} \mathrm{Al}$ and ${ }^{31} \mathrm{P}$ NMR experiments), but information on the non-periodic F subnetworks was only able to be obtained using NMR spectroscopy.

Although in many of the examples of NMR crystallography of microporous materials, the framework topology is often known in advance, for pure silica zeolites, it has been shown that rather than using NMR spectroscopy to "refine" structural models, structure "solution" is also possible. ${ }^{79,80}$ Initial work demonstrated that, using just basic information from diffraction (cell parameters and space group), a ${ }^{29} \mathrm{Si}$ MAS NMR spectrum and a series of Si-Si distance measurements (from two-dimensional homonuclear correlation experiments), it was possible to solve the framework structure of two zeolites (later identified as ITQ-4 and ferrierite). ${ }^{81}$ Figure 4 shows an overview of the structure determination strategy used, along with examples of the ${ }^{29} \mathrm{Si}$ MAS and two-dimensional correlation spectra of ITQ-4. A comparison of the framework structure derived using NMR and that from diffraction is also shown, demonstrating the good agreement achieved. In subsequent work, it was shown that by comparing experimental and calculated ${ }^{29} \mathrm{Si}$ chemical shift tensors (i.e., the isotropic and anisotropic components of the shielding interaction) improvement of the initial structural models was possible, with refinement of the exact atomic positions. ${ }^{79,82}$ Calculations were performed initially using small clusters, and gave good agreement with experiment for optimised structural models, ${ }^{79}$ but more recent work using a periodic GIPAW approach was shown to result in an improvement. ${ }^{82}$ In perhaps the most impressive advance in this area, the methods described above have been adapted and improved to enable structure determination in zeolites even when the space group is not known. ${ }^{80}$ While the unit cell parameters can 
usually be obtained even for XRD patterns with overlapping reflections, it can be considerably more difficult to determine the space group in this case. Although so far restricted to high-silica zeolites, these methods have demonstrated the power of NMR spectroscopy for structure solution, and offer great promise for future improvement and application to other types of microporous materials.

\subsection{Isotopic Enrichment}

The low natural abundance of many NMR-active isotopes (e.g., ${ }^{2} \mathrm{H}(0.012 \%),{ }^{17} \mathrm{O}$ $(0.037 \%)$ and $\left.{ }^{15} \mathrm{~N}(0.036 \%)\right)$ can hinder or preclude their routine study. However, in many cases, NMR spectra of these nuclei can provide valuable information on structure and dynamics in the solid state. Therefore, isotopic enrichment, which allows the acquisition of NMR spectra of these nuclei on a reasonable timescale, is often carried out, particularly for ${ }^{2} \mathrm{H}$, for which enrichment is generally comparatively cheap. In addition to uniform isotopic enrichment to enhance the overall signal, isotopic labelling may be used to provide sitespecific information ranging from the assignment of particularly complicated or unusual NMR spectra to information on the local structure and dynamics present in a specific location within the structure.

\section{${ }^{2} \mathrm{H}$}

${ }^{2} \mathrm{H}(\mathrm{I}=1)$ NMR is particularly useful for the study of dynamics, owing to the small (typically 100-250 kHz) first-order quadrupolar broadening of the resonances. The lineshapes are affected by motion on the $\mu$ s timescale (see Section 3.3), and variabletemperature experiments (carried out on either a static or rotating sample) can be used to extract information on the geometry and rate constant of any dynamic processes present. ${ }^{83}$ In addition, as the gyromagnetic ratio of ${ }^{2} \mathrm{H}$ is a factor of $\sim 6.5$ lower than that of ${ }^{1} \mathrm{H}$, homonuclear dipolar interactions are much weaker and ${ }^{2} \mathrm{H}$ MAS NMR spectra are typically of higher resolution. In heavily deuterated materials, the ${ }^{1} \mathrm{H}$ homonuclear spin 
network is broken into essentially isolated spins and the MAS NMR spectrum of the residual ${ }^{1} \mathrm{H}$ nuclei is of higher resolution than at natural abundance.

${ }^{2} \mathrm{H}$ (or D) enrichment is commonly achieved with the use of commercially-available deuterated starting materials (particularly $\mathrm{D}_{2} \mathrm{O}$ and the linkers of many common MOFs). For example, variable-temperature static ${ }^{2} \mathrm{H}$ NMR experiments have been used to observe ring flipping of deuterated terephthalate linkers, ${ }^{30,84,85}$ as shown for the $\mathrm{Sc}_{2} \mathrm{BDC}_{3} \mathrm{MOF}$, discussed above, in Figure 5(a). Gul-E-Noor et al. used deuterated trimesic acid as the linker in HKUST-1, removing the ${ }^{1} \mathrm{H}$ signal from the MOF framework and allowing the binding of adsorbed $\mathrm{H}_{2} \mathrm{O}$ to be probed by ${ }^{1} \mathrm{H} \mathrm{NMR}$, even at low levels of loading. ${ }^{86}$ The fact that $\mathrm{OH}$ and $\mathrm{NH}$ groups will exchange rapidly with OD and ND can be exploited as a means of introducing selective deuteration, and has allowed ${ }^{2} \mathrm{H}$ NMR to probe structural features such as hydrogen bonding at acid sites in zeolites. $4,5,7,8$

\section{${ }^{17} \mathrm{O}$}

Despite the ubiquitous presence of oxygen in MOFs and zeolites, ${ }^{17} \mathrm{O}(\mathrm{I}=5 / 2) \mathrm{NMR}$ is relatively underexploited, due to its poor sensitivity and the typically high cost of enrichment. However, as $\mathrm{O}$ atoms are often close to the active sites of microporous materials (e.g., Brønsted acid sites of zeolites and open metal centres in MOFs), ${ }^{17} \mathrm{O}$ NMR has great potential to provide information on both the structure of the active sites and any chemical processes occurring there. ${ }^{17} \mathrm{O}$ enrichment of zeolites may be achieved by heating the material in an atmosphere enriched in ${ }^{17} \mathrm{O}_{2}(\mathrm{~g})$ or $\mathrm{H}_{2}{ }^{17} \mathrm{O}(\mathrm{g})$. However, in many cases, chemically-inequivalent $\mathrm{O}$ species will exchange at different rates, leading to non-uniform enrichment. While non-uniform enrichment may not always be desirable, Stebbins et al. exploited the more rapid exchange of $\mathrm{Al}-{ }^{17} \mathrm{O}-\mathrm{Al}$ linkages in the natural zeolite, stilbite, to confirm their presence (in violation of Lowenstein's rule) in a series of hydrothermal enrichment and back-exchange experiments. ${ }^{87}$ The introduction of ${ }^{17} \mathrm{O}$ into AlPOs, using just a small volume $(50 \mu \mathrm{l})$ of $35 \%$-enriched $\mathrm{H}_{2}{ }^{17} \mathrm{O}(\mathrm{l})$ was achieved recently by Griffin et al., who added the small volume of water to a nominally-anhydrous ionothermal synthesis, 
and achieved overall enrichment levels in the resulting SIZ-4 of $\sim 4 \%{ }^{42}$ This represents a very cost-effective enrichment method (total cost of the $\mathrm{H}_{2}{ }^{17} \mathrm{O}$ used was $\subsetneq € 0.50$ ) and the enrichment level achieved was shown to be suitable both for DOR and two-dimensional MQMAS experiments (at 20.0 T), shown in Figures 5(b and c) for the as-made and calcined materials. One of the earliest examples of ${ }^{17} \mathrm{O}$-enriched MOFs is the work of Muller et al., who used ${ }^{17} \mathrm{O}$-enriched terephthalic acid and $\mathrm{H}_{2}{ }^{17} \mathrm{O}(\mathrm{l})$ to investigate the fate of $\mathrm{O}$ atoms in MOF-5 upon growth of $\mathrm{ZnO}$ nanoparticles in the pores. ${ }^{88}$ More recently, He et al. have developed procedures for the synthesis of some MOFs in a small volume of aqueous solvent to allow the use of reasonable volumes of $\mathrm{H}_{2}^{17} \mathrm{O}$ to be incorporated. ${ }^{89}$ It was shown that ${ }^{17} \mathrm{O}$ NMR of enriched UIO-66 was able to distinguish between oxo and hydroxide ligands at the $\mathrm{Zr}$ metal sites - a task that would be impossible for Bragg diffraction techniques. This ability to distinguish between $\mathrm{O}$ and $\mathrm{OH}$ is also of great interest when probing the Brønsted acid sites of zeolite (see also Section 3.3)..$^{4,7,7}$ In zeolites, the isotropic shift and quadrupolar coupling constant of ${ }^{17} \mathrm{O}$ can be used to distinguish between $\mathrm{Si}-\mathrm{O}-\mathrm{Si}$, Al-O-Si and Al-O-Al linkages, ${ }^{4,5,7,87}$ while the isotropic shift has been linked to the T-O-T (T $=\mathrm{Si}, \mathrm{Al}, \mathrm{P}$, etc.) angle. ${ }^{4,5,42}$ As there are typically many chemically similar, but magnetically distinct, $\mathrm{O}$ species present (even in a well-ordered zeolite), the resolution of individual species may not always be possible. However, Bull et al. demonstrated that, for the FAU and FER type siliceous zeolites ${ }^{11}$ (having no Al within the frameworks), the individual crystallographic $\mathrm{O}$ species could all be resolved by high-resolution MQMAS spectra and, in the latter case, multiple-field double rotation (DOR) spectra. ${ }^{41,90}$ More recently, Huo et al. demonstrated that ${ }^{1} \mathrm{H}^{-17} \mathrm{O}$ experiments are also capable of providing quantitative measurements of hydrogen bond lengths in mordenite..$^{50}$

\section{${ }^{13} \mathrm{C}$}

Carbon is ubiquitous in the linkers of MOFs, and most guest species in zeolites also contain carbon. While the natural abundance of ${ }^{13} \mathrm{C}(1.1 \%)$ is sufficient to allow routine study, enrichment of ${ }^{13} \mathrm{C}$ is often desirable to enhance the sensitivity of the experiment, allowing, for example, the detection of rare chemical species (i.e., catalytic intermediates or 
guests with low loading). ${ }^{91}$ In many cases, the desired ${ }^{13} \mathrm{C}$-enriched material is not commercially available and new synthetic procedures must be developed to prepare the material in a cost-effective manner, using the limited number of available ${ }^{13} \mathrm{C}$ sources.

In addition to the uniform enrichment of ${ }^{13} \mathrm{C}$, specific isotopic labels may be introduced. For example, Dawson et al. used selective isotopic enrichment of trimesic acid to confirm the counter-intuitive assignment of the resonances in the ${ }^{13} \mathrm{C}$ NMR spectra of the paramagnetic MOFs, HKUST-1 and STAM-1 (see Section 3.4). ${ }^{92}$ In the case of reactions catalysed by zeolites, mechanistic insight can be gained from so-called ${ }^{13} \mathrm{C}$ scrambling experiments, where specifically-placed ${ }^{13} \mathrm{C}$ labels are redistributed throughout the molecule in question. ${ }^{91,93}$

${ }^{15} N$

While ${ }^{14} \mathrm{~N}$ has high $(99.964 \%)$ natural abundance, its integer spin $(\mathrm{I}=1)$ and low gyromagnetic ratio make ${ }^{14} \mathrm{~N}$ NMR spectra typically very challenging to acquire. Therefore, ${ }^{15} \mathrm{~N}$ enrichment is often carried out simply to increase the sensitivity of this $\mathrm{I}=$ $1 / 2$ isotope, allowing high-resolution spectra to be recorded with conventional pulse sequences. ${ }^{15} \mathrm{~N}$ NMR enrichment is used extensively in zeolites, where enriched "probe" molecules, such as $\mathrm{NH}_{3}$, and other amines can be used to investigate the strength of acid sites. ${ }^{91,94}$ In addition, as discussed below in Section 3.3, Holland et al. ${ }^{95}$ used ${ }^{15} \mathrm{NH}_{3}$ to identify the binding sites of $\mathrm{NH}_{3}$ in zeolite $3 \mathrm{~A}$. While ${ }^{15} \mathrm{~N}$ NMR spectra of MOFs are typically carried out at natural abundance, an unusual consequence of ${ }^{15} \mathrm{~N}$ enrichment was demonstrated by Morris et al. ${ }^{96}$ who showed that rapid $\mathrm{T}_{1}$ and $\mathrm{T}_{2}$ relaxation of ${ }^{1} \mathrm{H}$ and ${ }^{13} \mathrm{C}$ in the MOF, IRMOF-3, was induced by a dipolar-quadrupolar cross-coupling involving the large quadrupolar interaction of ${ }^{14} \mathrm{~N}$ in the 2-aminoterephthalate linkers. By enriching in ${ }^{15} \mathrm{~N}$, this effect was removed, allowing ${ }^{1} \mathrm{H}$ and ${ }^{13} \mathrm{C}$ NMR spectra with higher resolution to be recorded. Therefore, ${ }^{15} \mathrm{~N}$ enrichment has the potential to enhance the resolution of NMR spectra of many N-based MOFs, particularly for the metal centres in many MOFs where the first coordination sphere of the metal is N-rich (e.g., imidazolate- and cyanide-based 
MOFs). In addition, Kong et al. recently carried out ${ }^{15} \mathrm{~N}^{13} \mathrm{C}$ REDOR experiments for ${ }^{15} \mathrm{~N}$ enriched linkers (in combination with selective ${ }^{13} \mathrm{C}$-labelling) in mixed-linker MOFs, based on the MOF-5 topology. ${ }^{51}$ These experiments allowed the mesoscale spatial distribution of the different linkers to be probed, and it was shown that different distributions (e.g., random, clustering and alternating) could readily be distinguished.

\section{${ }^{29} \mathrm{Si}$}

The enrichment of zeolites with ${ }^{29} \mathrm{Si}$ has long been known, and is relatively straightforward, with commercially-available ${ }^{29} \mathrm{Si}\left(\mathrm{OC}_{2} \mathrm{H}_{5}\right)_{4}$ or ${ }^{29} \mathrm{SiO}_{2}$ typically used in place of the natural-abundance $\mathrm{Si}$ precursor. While the natural abundance of ${ }^{29} \mathrm{Si}(4.69 \%)$ is sufficient to allow one-dimensional spectra to be recorded on a reasonable timescale, more informative two-dimensional spectra (where the transfer of magnetisation between NMRactive species is exploited) may require enrichment. For example, early work by Fyfe et al. showed that it was possible to use two-dimensional correlation spectroscopy (COSY) experiments to determine the framework connectivities present in the siliceous zeolite, ZSM-39, which contains 5 distinct Si species and 128 connections between inequivalent $\mathrm{Si}^{4,5,97}$ More recently, ${ }^{29} \mathrm{Si}$ enrichment work has been focused more on the observation of scarce zeolite precursor species formed in the early stages of the synthesis of zeolites. For example, Haouas and Taulelle ${ }^{98}$ were able to identify several precursor building units in a precursor solution enriched to $19 \%{ }^{29} \mathrm{Si}$, using two-dimensional INADEQUATE experiments.

\subsection{Characterisation of Host-Guest Interactions}

The utility of microporous materials lies in their ability to selectively absorb and interact with guest molecules, and to act as catalysts for specific reactions. ${ }^{1}$ Guests can include the templates present in as-made materials, as well as gases and a range of other small- to medium-sized molecules, e.g., alkanes or drugs. Guest species may covalently bind directly to the framework (as is often observed in many MOFs), interact with the 
framework through hydrogen bonding, or alternatively, may be found "free" in the pores. NMR spectroscopy offers an ideal probe for the investigation of host-guest interactions, as it is able to study both frameworks and guests, and to detect the changes (in structure and dynamics) in both upon interaction and/or binding. Typically, the symmetry of the "empty" framework is reduced upon the uptake of guest species: an effect readily seen in the NMR spectrum by an increase in the number of peaks observed. The number of peaks from the guest molecules themselves provides information on the number of binding sites present, while changes to the spectral lineshapes (particularly with variable-temperature experiments, as described above) also provide information on the type of binding occurring, i.e., by probing motional behaviour.

Although perhaps not a "guest molecule" in the conventional sense, the interaction between microporous frameworks and the inert gas, Xenon, provides a useful probe of the topology and structure of porous solids. ${ }^{10,99}{ }^{129} \mathrm{Xe}(\mathrm{I}=1 / 2)$ exhibits an extensive chemical shift range, with the size and shape of the pores or channels in which the Xe is found, interactions between Xe molecules, the presence of paramagnetic species and the presence of other guest molecules, all affecting the spectra observed. In many cases, the sensitivity of ${ }^{129}$ Xe NMR spectroscopy can be significantly improved by optical pumping using a laser, resulting in hyperpolarised $\mathrm{Xe}^{100}$ enabling materials with low $\mathrm{Xe}$ concentration to be studied. When low concentrations are used, the main contribution to the ${ }^{129} \mathrm{Xe}$ chemical shift is then purely the interaction between the Xe and the framework, easing spectral analysis. There is a significant difference (of $\sim 100-300$ ppm) in shift between free Xe gas and $\mathrm{Xe}$ trapped within the pores, as seen in Figure $6(\mathrm{a}),{ }^{129} \mathrm{Xe}$ NMR spectra of hyperpolarised Xe in IRMOF-8 (a Zn-based MOF with naphthalenedicarboxylate linkers). ${ }^{101}$ In general (for low Xe concentrations), the larger the pores the lower the Xe shift (i.e., closer to that of the free gas). More restricted Xe typically has a higher shift, and can also exhibit anisotropic (powder pattern) lineshapes as a result of the interaction with the framework. Figure 6(a) shows that at $221 \mathrm{~K}$ two distinct peaks are observed, demonstrating the presence of two different Xe environments in loaded IRMOF-8 (thought 
to be related to adsorption in the $\alpha$ and $\beta$ cages, respectively). ${ }^{101}$ However, the isotropic lineshapes that are observed indicate that within each cage the Xe is rapidly exchanging between different binding sites, averaging any anisotropy. Exchange processes can also affect the observed chemical shift of a Xe resonance (particularly if there is any exchange with free Xe gas) and can be monitored either using variable-temperature experiments (and studying the changes in linewidth or lineshape) or using two-dimensional exchange spectroscopy (EXSY) experiments, where the intensity of cross peaks as a function of mixing time provides information on the exchange rate. For the example described above, EXSY experiments carried out at $221 \mathrm{~K}$ reveal that exchange between the two Xe binding sites occurs, with an exchange rate of $\sim 10 \mathrm{~s}^{-1} .{ }^{101}$ For more information on the use of Xe NMR to probe the structure of microporous materials see the examples discussed in Refs. 5 and 6.

One of the most ubiquitous "guest" molecules is water, with many microporous materials, particularly phosphates and MOFs, susceptible to hydration when in their active (i.e., calcined or empty) forms. Significant changes can be seen in the NMR spectrum, e.g., $6 \mathrm{Al}$ and $6 \mathrm{P}$ resonances are observed for hydrated AlPO-34 (CHA type framework) in contrast to the single resonances found in both NMR spectra for the calcined material. ${ }^{102}$ In addition, 5 of the 12 water molecules incorporated into the fully hydrated form of AlPO-34 coordinate to the $\mathrm{Al}$ cations, resulting in $\mathrm{Al}(\mathrm{IV})^{\prime} \mathrm{Al}(\mathrm{V})$ and $\mathrm{Al}(\mathrm{VI})$ resonances. $^{102}$ Binding, or even close proximity of water to the framework, may have dramatic influence on the NMR parameters, notably with chemical shift changes and changes to the quadrupolar coupling, possibly requiring tailored experiments for observation. Variation of the water content in as-made or loaded materials is generally rarer, although, as discussed above, in situ ${ }^{27} \mathrm{Al}$ and ${ }^{31} \mathrm{P}$ NMR spectroscopy was used to follow the phase transformation of AlPO-53(A) to JDF-2, a related, but anhydrous, material. ${ }^{33}$ In this case the dehydration was driven by the frictional heating of the sample under MAS, and could be easily reversed by leaving the sample standing in air. 
Guest molecules in porous solids can be investigated using ${ }^{1} \mathrm{H},{ }^{13} \mathrm{C}$ and ${ }^{15} \mathrm{~N}$ NMR spectroscopy, which can identify the molecules incorporated (of particular importance when materials are exposed to multiple guests simultaneously), and determine the number and type of binding sites. The typical experiments used range from simple MAS and CP MAS, to two-dimensional experiments (including single-quantum/singlequantum and double-quantum/single-quantum correlations), and experiments where interactions between the guest and framework are exploited (thereby locating the binding sites within the structure). As an example, Figure 6(b) shows that two different $\mathrm{NH}_{4}^{+}$ environments (termed I and II) in zeolite 3A loaded with ammonia were identified using ${ }^{15} \mathrm{~N}$ MAS and CP MAS experiments. ${ }^{95}$ The difference in CP efficiency (and hence the molecular mobility) for the two sites was used to determine the strength of the binding in each case, with the ammonia in site I found to be more strongly bound than that in site II. Spectra recorded at different loading levels indicated that site I was occupied preferentially at lower loadings, and double resonance $\left({ }^{15} \mathrm{~N} /{ }^{23} \mathrm{Na}\right.$ TRAPDOR) experiments showed a strong interaction between nitrogen and the $\mathrm{Na}^{+}$cation, and so must be located in the six-membered ring of the zeolite structure, as shown in Figure 6(b). ${ }^{95}$

Many microporous frameworks can be used for the selective absorption of gases. In some cases, one gas absorbs preferentially, or for more complex materials different gases may absorb in different parts of the structure. For example, during the synthesis of the $\mathrm{Cu}$ based MOF STAM-1, the initial trimesate linker undergoes selective monoesterification, producing a porous solid with two different types of channels, with hydrophilic and hydrophobic surfaces. ${ }^{24}$ The two have very different adsorption properties, exhibiting significant differences in the kinetics of adsorption of $\mathrm{O}_{2}$ and $\mathrm{N}_{2}$, enabling the separation of these gases from air. Furthermore, for some molecules, the site of adsorption can be "switched" between the two channels by changing the conditions used. In other recent work, Kong et al. used ${ }^{13} \mathrm{C}$ NMR spectroscopy to investigate ${ }^{13} \mathrm{CO}_{2}$ adsorbed in a $\mathrm{Mg}$ form of CPO-27 (a MOF with 2,5-dihydroxyterephthalic acid linkers, displaying excellent $\mathrm{CO}_{2}$ capture properties). ${ }^{103}{ }^{13} \mathrm{C}$ NMR spectra (shown in Figure 6(c)), acquired between 
temperatures of 200 and $380 \mathrm{~K}$ exhibit a distinct CSA powder pattern, indicative of a strong interaction between the $\mathrm{Mg}$ and the $\mathrm{CO}_{2}$, and of restricted molecular motion. The axially-symmetric lineshape observed is consistent with an end-on (but non-linear) coordination of $\mathrm{CO}_{2}$ and with rotation around the $\mathrm{Mg}-\mathrm{OCO}$ vector, as shown in Figure 6(c). As the temperature decreases a broadened lineshape is observed (at $150 \mathrm{~K}$ ), indicating an intermediate motional regime as rotation slows. When the temperature decreases further to $100 \mathrm{~K}$, the lineshape resembles that expected for rigid $\mathrm{CO}_{2}$. In Ref. 103, relaxation measurements were also used to determine the correlation time and activation energy for $\mathrm{CO}_{2}$ rotation. The experimental measurements were able to provide restraints in DFT and molecular dynamics calculations. ${ }^{103}$

One particularly important property of MOFs is their high degree of flexibility, either when heated, or upon the adsorption of guest species. A well-studied family of MOFs are the terephthalate-based MIL-53 materials (known with Al, Sc, Ga, Cr and Fe metal cations). In particular, a number of phase transitions has been demonstrated for MIL-53(Al), ${ }^{104}$ with an as-made form where free terephthalate linker molecules occupy the channels, a high-temperature form where the pores are empty and a room-temperature form where the channels contain water, with channel sizes of $7.3 \times 7.7 \AA^{2}, 8.5 \times 8.5 \AA^{2}$ and $2.6 \times 13.6 \AA^{2}$, respectively). ${ }^{104}$ This considerable change in pore size is often referred to as "breathing". All three forms of MIL-53(Al) were characterised using ${ }^{1} \mathrm{H},{ }^{13} \mathrm{C}$ and ${ }^{27} \mathrm{Al} \mathrm{NMR}$, confirming the presence and/or absence of guest molecules, and demonstrating that the phase transition upon hydration is driven by the trapped water molecules interacting with the carboxylate groups through hydrogen bonding. ${ }^{104}$ The Sc-based form of MIL-53 also exhibits a series of phase transitions, which can be followed using NMR spectroscopy the effect of hydration upon the ${ }^{45}$ Sc NMR spectrum was shown previously in Figure 2(c). ${ }^{34}$ The utility of MIL-53 MOFs for drug delivery has also been recently demonstrated. ${ }^{105}$ It was shown by Férey and co-workers that MIL-53(Cr, Fe) adsorb 20 wt \% of ibuprofen, also causing significant pore expansion. The nature of the drug-framework interaction was studied using ${ }^{1} \mathrm{H}$ and ${ }^{13} \mathrm{C}$ solid-state NMR experiments, although spectral analysis is 
complicated by the paramagnetic nature of $\mathrm{Cr}$ and Fe. NMR spectroscopy was able to confirm the presence of ibuprofen in each loaded material and determine that it was adsorbed in its neutral form. Consideration of the ${ }^{1} \mathrm{H}$ and ${ }^{13} \mathrm{C}$ chemical shifts of the loaded drug confirmed that it adopted a conformation close to that of its pure form - a conclusion that was also supported by complementary DFT calculations. ${ }^{105}$ For the materials studied, slow and complete delivery of the ibuprofen was achieved under physiological conditions after three weeks.

One advantage of NMR spectroscopy over diffraction-based methods is its sensitivity to dynamics over a range of motional timescales. Fast motion can be detected using changes in relaxation rates, and slow motion by two-dimensional exchange experiments. Motion on timescales intermediate to these extremes can also be probed by studying the changes in lineshape and linewidth of the anisotropically-broadened lines present in NMR spectra of solids. The timescales that can be probed by this type of lineshape analysis are determined by the inverse of the coupling/interaction perturbed. As mentioned in the previous section, ${ }^{2} \mathrm{H}(\mathrm{I}=1)$ NMR spectroscopy has been used extensively for the study of motion in solids over many years. ${ }^{83}$ This approach is also of use for studying the dynamic behavior of organic guest molecules within the pores of microporous solids. Comparison of experimental lineshapes (often acquired as a function of temperature) to those from simulation is able to provide information both on the rate and type of dynamic process. Many early examples focused on the study of loaded zeolites, ${ }^{5}$ including, more recently, the study of methanol- $\mathrm{d}_{4}$ within $\mathrm{NaX}$ and $\mathrm{NaY}$ zeolites. ${ }^{106}$ In this latter example, variable temperature NMR was able to identify gas phase, liquid-like and immobilised methanol in both zeolites over a temperature range of 20 to $300 \mathrm{~K}$. Two modes of methanol binding were identified - one involving interaction with the $\mathrm{Na}^{+}$cation and the second an interaction with the framework $\mathrm{O}$ in Al-O-Si linkages. From ${ }^{2} \mathrm{H}$ relaxation measurements there was also evidence for the formation of small clusters (usually trimers) of methanol within the pores. ${ }^{106}$ More recent work has involved the study of MOFs, including a study by Ueda et al. of deuterated cyclohexane 
confined in IRMOF-1, which found a number of different motional regimes as a function of temperature, ${ }^{107}$ and work by Wright and co-workers on deuterated benzene incorporated into MOF-5 (IRMOF-1), where the incorporated molecules exhibit rotation around their $\mathrm{C}_{6}$ axis at lower temperature, but isotropic motion as the temperature increases. $^{84}$

Motion of guests within the framework can also be detected indirectly, i.e., by the effect this causes upon the framework. Recent work by Antonijevic et al. used ${ }^{27} \mathrm{Al}$ NMR spectroscopy to detect the presence of dynamics in AlPOs (specifically in AlPO-14 with isopropylammonium and piperidinium cations within the pores). ${ }^{46}$ This was probed using variable-temperature ${ }^{27} \mathrm{Al}$ STMAS experiments, as shown in Figure 6(d). The MQMAS spectrum shows four sharp resonances for the four distinct Al species in the material. However, for STMAS, the satellite transitions used in the experiment are broadened by the large first-order quadrupolar interaction, and any motion on the $\mu$ s timescale results in a broadening of the spectral lineshapes. ${ }^{45,46}$

In addition to their uses for storage and separation, porous solids also have great potential as catalysts. ${ }^{7,8,10}$ While the use of MOFs in this area is in its infancy, zeolites (and zeotypes) have been widely used, particularly in the petrochemical industry, where they have largely replaced liquid acid catalysts. The most prominent examples are the aluminosilicate zeolites, owing to their high thermal stability, well-defined local geometry and the strength and/or selectivity of their acid sites. The performance of such catalysts is determined by the distribution, type and strength of the acid sites, and NMR spectroscopy is well placed both to investigate this, and to follow the chemical reactions that can be carried out. ${ }^{7,810}$ Catalysis may occur at Brønsted sites, e.g., Al-OH-Si in zeolites or M-OH-P in phosphates, or at Lewis sites (generally at under-coordinated cations that can accept electrons). It is possible to discern information about the type of acid sites present simply from the ${ }^{1} \mathrm{H}$ chemical shift - with e.g., shifts of -0.5-1.5 ppm typical of Metal-OH groups in large channels, 1.2-2.2 ppm Si-OH groups on external surfaces, 2.4-3.6 ppm for non- 
framework Al-OH groups, 3.6-4.3 ppm for Brønsted Si-OH-Al in larger channels and 4.6$8.0 \mathrm{ppm}$ for Si-OH-Al in smaller channels. In all cases, an increase in hydrogen bonding strength increases the chemical shift. ${ }^{8}$ Information can also be obtained from other nuclei, with the presence of a Brønsted acid ${ }^{1} \mathrm{H}$ increasing the ${ }^{27} \mathrm{Al}$ quadrupolar interaction, while double-resonance experiments such as TRAPDOR can be used to probe Al-H interactions and spatial proximities. ${ }^{108}$ Another approach to characterising acid sites is to use probe molecules, i.e., small molecules that bind to or interact with the acid sites, changing their local structure and, therefore, their NMR spectra. For example, a linear correlation of the ${ }^{1} \mathrm{H}$ chemical shift of the pyridinium ions formed when pyridine (usually ring deuterated) adsorbs onto an acid site is observed with increasing Brønsted acid strength, ${ }^{7,8109}$ with shifts from 12-20 ppm exhibited. Alternatively, the ${ }^{13} \mathrm{C}$ chemical shift of the carbonyl in acetone is also very sensitive to the presence of acid sites, with higher shifts observed for more acidic sites. ${ }^{7,8,110}$

Characterising the position, strength, type and distribution of acid sites is the first (and perhaps most vital) step in understanding the catalytic properties of a material. However, NMR is also able to provide real insight into the catalytic reactions that can be carried out by following the amount and type of products/reactants present. This can be done in so-called "batch mode" where reactions are carried out, sometimes in sealed glass ampules that can fit directly into an MAS rotor, at different conditions (temperatures/times) before being quenched and studied using $\mathrm{NMR}^{7.8}$ In a recent example, the methanol-to-olefin conversion catalysed by low silica AlPO-34 was studied using ${ }^{1} \mathrm{H}$ and ${ }^{13} \mathrm{C}$ NMR spectroscopy. ${ }^{111}$ The catalytic properties were attributed to Brønsted acid sites, arising from the substitution of $\mathrm{Si}$ for $\mathrm{P}$ which in turn leads to a negative framework charge that is then compensated by hydroxyl protons. ${ }^{13} \mathrm{C}$ NMR identified a lower formation of aromatic molecules than using SAPO-34, but much greater formation of dienes. ${ }^{111}$ True "in situ" measurement, while in principle much more informative, is also considerably more complicated, requiring advanced probe and reactor hardware - see Section 4 for more detail. 


\subsection{Paramagnetic Systems}

Many of the most interesting microporous materials are paramagnetic owing to, e.g., transition metal ions substituted into zeolitic frameworks or present within the SBUs of MOFs. The unpaired electrons have both through-bond and through-space interactions with the nuclei. ${ }^{112}$ Where present, the through-bond hyperfine interaction typically dominates the spectrum, leading to isotropic shifts and shift anisotropies hundreds of times greater than in diamagnetic materials. The through-space pseudocontact interaction is typically smaller, but longer range (up to several $\mathrm{nm}$ ) than the hyperfine interaction and can also give isotropic shifts and shift anisotropies. In addition, coupling of the magnetic dipole moment of the electron (which relaxes very rapidly on the nuclear timescale) can lead to enhancement of nuclear $T_{1}$ and $T_{2}$ relaxation, both of which are often on the scale of ms, or faster in paramagnetic materials. The bulk magnetic susceptibility (BMS) is also responsible for anisotropic broadening, but modelling this is not trivial, and requires detailed knowledge of the crystallite size and shape. Therefore, this is often neglected in the analysis of NMR spectra of paramagnetic materials.

The additional interactions with the unpaired electrons can complicate the acquisition of solid-state NMR spectra by conventional approaches. However, there are several strategies that may be applied in such cases to obtain relevant information from solid-state NMR. In the case of a series of paramagnetic MeAPOs, Mali et al. ${ }^{113}$ used a static spin-echo mapping experiment, with correction of each spin echo for different $\mathrm{T}_{2}$ relaxation across the lineshape, as shown schematically in Figure 7(a), to demonstrate that the ${ }^{31} \mathrm{P}$ resonances were too broad (up to $\sim 2.2 \mathrm{MHz}$ at $\mathrm{B}_{0}=7.1 \mathrm{~T}$ ) to be recorded under MAS (a well-known phenomenon in some paramagnetic materials, where the width of individual spinning sidebands in the MAS NMR spectrum exceeds their separation and no signal is observed). The ${ }^{31} \mathrm{P}$ NMR spectra, shown in Figure $7(b)$, confirmed that the transition metal (Me) ions had been incorporated into the MeAPO frameworks (rather 
than being located in the pores). Quantitative information on the bulk average Me : P ratio of the materials was also obtained, as well as information about the distribution of the Me ions within the framework. ${ }^{113}$

It is interesting to note that the use of broadband excitation pulses has not yet been extended to paramagnetic materials, and spectral mapping techniques still use narrowband $(\sim 100 \mathrm{kHz})$ square pulses. However, it can be appreciated that in cases where $\mathrm{T}_{1}$ relaxation constants may be on the order of a few ms, the application of long ( 0.05 ms) pulses will lead to significant relaxation-related losses, particularly as the pulses generally rely on very specific manipulation of the spins throughout the duration of the frequency sweep. However, one sensitivity-enhancing method that has been shown to be effective in some paramagnetic materials is the CPMG pulse sequence, ${ }^{63,64}$ in which the magnetisation is repeatedly refocused by a series of spin echoes. For example, Kervern et al. showed for small $\mathrm{Tb}^{3+}$ complex that, despite a very short apparent ${ }^{1} \mathrm{H} \mathrm{T}_{2}$ (on the order of tens of $\mu$, measured from the MAS linewidths), $\mathrm{T}_{2}$ relaxation constants of 1.7 and $7.5 \mathrm{~ms}$ could be measured using CPMG, with a corresponding enhancement of the signal-to-noise ratio. ${ }^{114}$ In cases such as this, where Curie relaxation is absent, therefore, CPMG may be a very useful sensitivity enhancement approach, particularly for nuclei with lower natural abundance, such as ${ }^{13} \mathrm{C}$.

High-resolution NMR spectra of some paramagnetic materials may be obtained, although typically using very different acquisition approaches than would be used for most diamagnetic materials. Owing to the large anisotropy and shift dispersion often present for paramagnetic materials, the use of lower field (where the shift dispersion may be only a few hundred $\mathrm{kHz}$ and resonances may be acquired in very few steps) could be advantageous. In addition, rapid MAS (typically $>40 \mathrm{kHz}$ ) has been used to reduce the number and intensity of the spinning sidebands in the NMR spectra of paramagnetic materials. Some of the earliest examples of high-resolution NMR spectra of paramagnetic microporous materials were the ${ }^{13} \mathrm{C}$ NMR spectra of the Cr-based MOF, MIL-101, loaded 
with ibuprofen ${ }^{115}$ and the Cu-based MOF, HKUST-1. ${ }^{116}$ These spectra were recorded with relatively slow MAS (between 7 and $20 \mathrm{kHz}$ ) and, particularly in the spectrum of HKUST1 recorded by Peterson et al., ${ }^{116}$ there is a significant background signal, which dwarfs the resonances arising from the MOF itself. In the spectrum of Horcajada et al., ${ }^{115}$ the resonances from ibuprofen were observed, as discussed above, whereas only one resonance attributed to the MIL-101 framework was present (at $175 \mathrm{ppm}$ ). The work of Gul-E-Noor et al., ${ }^{117}$ also on HKUST-1, used a suppression sequence to remove the ${ }^{13} \mathrm{C}$ background signal, but the relatively low MAS rate $(10 \mathrm{kHz})$ led to a spectrum that was still cluttered with spinning sidebands. However, two resonances could be identified, at 228 ppm (assigned to the $\mathrm{CH}$ and quaternary $\mathbf{C}$ of the trimesate linker) and at $-86 \mathrm{ppm}$ (assigned to the $\mathrm{Cu}$-bound $\mathrm{CO}_{2}$ ). The position of this latter resonance was observed to vary between -56 and $-86 \mathrm{ppm}$, for the fully-hydrated and fully-dehydrated MOFs, respectively. More recently, Dawson et al. ${ }^{92}$ recorded the ${ }^{13} \mathrm{C}$ NMR spectra of the two MOFs, HKUST-1 and STAM-1, using very fast $(60 \mathrm{kHz})$ MAS and a spin-echo pulse sequence both to remove the background signal and to refocus any very broad resonances. In addition, as shown in Figure 7(c), frequency-stepped acquisition was used to reveal three ${ }^{13} \mathrm{C}$ resonances for HKUST-1 and seven for STAM-1 (in contrast to the four reported previously by Mohideen et al. ${ }^{24}$ who used slower $(12.5 \mathrm{kHz})$ MAS and cross polarisation from ${ }^{1} \mathrm{H}$ ). The additional resonance for HKUST-1 was observed at $\sim 850 \mathrm{ppm}$, and had a full width at half maximum of $10 \mathrm{kHz}$, explaining why it was not observed in earlier studies. Resonances for STAM-1 were also observed at $~ 850,227$ and -50 ppm, as well as the four reported by Mohideen et al., at 181, 178, 174 and 50 ppm. Despite an even larger shift dispersion (resonances between 1434 and $140 \mathrm{ppm}$ ), de Combarieu et al. ${ }^{118}$ were able to observe the ${ }^{13} \mathrm{C}$ MAS NMR spectrum of Fe-MIL-53 loaded with benzoquinone without frequency stepping, owing to the use of a low-field (4.7 T) spectrometer. All resonances expected for the benzoquinone were observed between 140 and 281 ppm, as well as four resonances assigned to the terephthalate linker of the MOF. 
The assignment of the ${ }^{13} \mathrm{C}$ NMR spectra of paramagnetic MOFs is typically made following the intuitive approach that resonances with larger paramagnetic contributions to their shifts and linewidths will arise from $C$ species closer to the paramagnetic centres. However, Dawson et al. ${ }^{92}$ prepared samples of HKUST-1 and STAM-1 from selectively ${ }^{13} \mathrm{C}$ labelled trimesic acids, and demonstrated that, when the acid group of the linker was labelled, the resonance at $-50 \mathrm{ppm}$ (and that at $178 \mathrm{ppm}$ in STAM-1) increased in relative intensity, whereas labelling the benzene ring led to an increased relative intensity of the resonances at 850 and 227 ppm (and those at 181 and 174 ppm in STAM-1), as shown in Figure $7(\mathrm{~d})$. This shows that the simplistic, intuitive approach cannot be relied upon for the assignment of NMR spectra of paramagnetic materials, even in the seemingly simple case of HKUST-1, which only contains three crystallographically- and chemically-distinct C species.

\section{Summary and Future Directions}

In general, solid-state NMR experiments and protocols have now matured to the stage where characterisation of the structure, disorder and dynamics of many microporous materials is possible. While the presence of paramagnetic ions or nuclei with very low sensitivity or very large quadrupolar broadening may require some further experimental developments (e.g., more efficient two-dimensional spectra with very short mixing times, more routine access to higher $B_{0}$ field strengths and faster MAS rates, improved bandwidth for wideline excitation, etc.,), the real future advances in solid-state NMR of microporous materials will come from the development of entirely new hardware, allowing access to completely different experimental regimes.

While some further sensitivity gains may be achieved through the development of new composite pulses (particularly for high-resolution NMR spectra of quadrupolar nuclei), the future of sensitivity-enhanced NMR experiments appears to lie with dynamic nuclear polarisation (DNP) experiments, ${ }^{10,19}$ in which microwave irradiation creates non- 
equilibrium magnetisation on unpaired electrons typically on radicals, which is then transferred to ${ }^{1} \mathrm{H}$ and, from there, to the nucleus of interest. This results in a large polarisation enhancement of the nucleus of interest, with very high (but highly experiment-dependent) enhancement factors reported in the literature. While much work is still required to develop reliable protocols for the introduction of radical species into the pores of microporous materials with the minimum perturbation to their structure and activity, Blanc et al. ${ }^{120}$ recently demonstrated the use of DNP on the ${ }^{13} \mathrm{C}$ and ${ }^{15} \mathrm{~N}$ NMR spectra of a series of microporous organic polymers, where the signal enhancement allowed ${ }^{13} \mathrm{C}$ CP MAS NMR spectra to be recorded in $10-43 \mathrm{~min}$, and ${ }^{15} \mathrm{~N}$ CP MAS NMR spectra in $0.8-14 \mathrm{~h}$ (depending on the concentration of the $\mathrm{N}$-containing functional groups). Furthermore, Pourpoint et al. ${ }^{121}$ recently used DNP-enhanced recoupling experiments to measure Al-C distances in MIL-100(Al) with an experimental time of just 7 h. One advance in DNP that would be particularly advantageous for microporous materials is the development of tuneable microwave sources, which would be able to exploit the inherent unpaired electrons of paramagnetic MOFs and MeAPOs, rather than requiring the addition of radicals to the system.

The development of MAS hardware capable of operating under extremely low or high temperatures, and under elevated pressures, represents an important progression of MAS NMR experiments towards the ultimate goal of being able to study microporous materials under realistic operating conditions for catalysis. While variable temperature MAS experiments are possible (including the cold-sample MAS pioneered by Levitt ${ }^{122}$ and commercially-available laser-heated MAS probes $^{123}$ ), the application of variabletemperature and high-pressure MAS remains a considerable technical challenge, particularly when a number of phases are involved in the reaction. Very recent work by Lercher and co-workers, describing the design of a microautoclave MAS NMR rotor capable of pressures of up to 20 bar and temperatures of up to $200{ }^{\circ} \mathrm{C}$ at MAS rates of $2-3$ $\mathrm{kHz}$, shows the potential such experiments will have. ${ }^{124}$ In this work, in situ studies of the kinetics and mechanism of the reaction of cyclohexanol on zeolite HBEA were studied at 
$130{ }^{\circ} \mathrm{C}$, with a series of ${ }^{13} \mathrm{C}$ MAS spectra acquired over a period of 23 hours, following the reactant loss and product formation. While the development of MAS hardware capable of studying catalytic reactions in situ (i.e., with a continuous reactant/product flow) began in the late 1990s, the MAS rates achievable are currently limited to $\sim 2-4 \mathrm{kHz}$, and no such hardware is commercially available. ${ }^{8}$ However, despite these technological limitations, much invaluable information on catalysed reactions can be obtained from in situ MAS NMR experiments, including identification of intermediates or transition states, the mobility of species within a catalyst bed, and the kinetics of the reaction(s) occurring. ${ }^{125-127}$ One interesting recent example of the extension of solid-state NMR experiments to the study of catalytic systems is the "operando" experiments of Roberts et al., ${ }^{128}$ in which ${ }^{1} \mathrm{H}$ NMR spectra of a (static) catalyst bed were recorded at different time points during the oligomerisation of ethane, allowing the conversion and product distribution to be followed with time. Further development of such MAS rotors and probes would be of great advantage when investigating the mechanisms of catalysed reactions.

In addition to experimental advances, which promise to provide ever more information-rich data, the constant advance of computing hardware and improvements to codes suitable for studying solids (particularly crystalline, periodic systems) will allow more detailed interpretation of the data. Of particular interest for microporous materials is the improvement of codes capable of calculating NMR parameters such as J couplings and paramagnetic interactions in solids, as well as codes able to calculate meaningful NMR parameters from (typically very large) molecular dynamics calculations, ${ }^{6,9,10,27}$ allowing effects such as motion and finite temperature to be taken into account. In addition, the increased use of dispersion correction schemes and the introduction of relativistic corrections will improve the accuracy of calculations.

As discussed above, solid-state NMR spectroscopy, particularly in combination with other experimental (e.g., crystallography) and computational (e.g., DFT) techniques, can offer great insight into both the local and longer-range structures of microporous 
materials. Such information is vital to the understanding of the function of these materials and it is to be hoped that further developments, particularly of hardware capable of accessing conditions currently unavailable for high-resolution NMR experiments, will, over the coming years, continue to increase the information provided by solid-state NMR spectroscopy.

\section{Acknowledgements}

SEA thanks the Leverhulme Trust (F/00 268/BJ) for funding. Mr Scott Sneddon is gratefully acknowledged for assistance with the preparation of this manuscript. 


\section{Biographies}

Sharon Ashbrook is a Professor in Physical Chemistry at the University of St Andrews. She obtained her DPhil from Oxford (2000) and was awarded a Royal Society Dorothy Hodgkin Research Fellowship in 2003, which she held in Cambridge. She has 105 publications and has been awarded the RSC Harrison Prize (2004) and RSC Marlow Award for Physical Chemistry (2011), as well as the Royal Society of Edinburgh

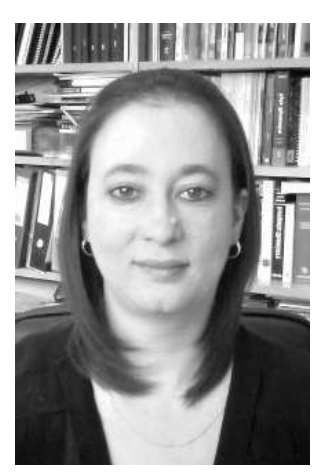
Makdougall Brisbane Medal for Physical Science (2012). Her research combines high-resolution multinuclear NMR spectroscopy with periodic first-principles calculations to investigate structure, order and dynamics in the solid state.

Daniel Dawson manages the EaStCHEM solid-state NMR facility at the University of St Andrews. He obtained his MChem from the University of Liverpool in 2009 and his PhD from the University of St Andrews in 2014. His current research interests include NMRbased studies of host-guest interactions in paramagnetic MOFs and characterisation of phase transformations occurring in microporous

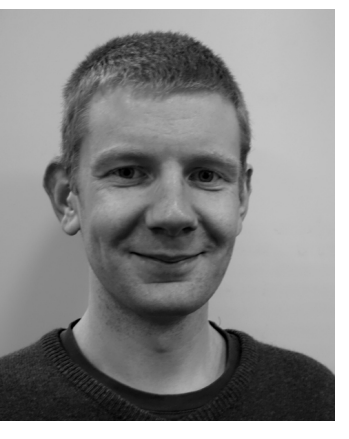
metallophosphates.

Valerie Seymour graduated with a MChem from Cardiff University in 2008. She then moved to the University of St Andrews where she completed a thesis entitled "Multinuclear Solid-State NMR for the Characterisation of Inorganic Materials", graduating in 2013. Since 2012, Valerie has continued at the University of St Andrews as a postdoctoral researcher. Her research focuses on the application of

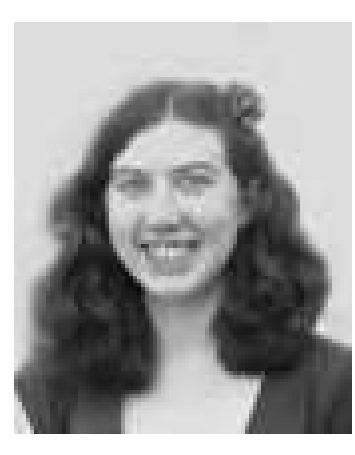
multinuclear solid-state NMR techniques, often in combination with first-principles DFT calculations, to investigate microporous framework materials. 
Table 1. Summary of the nuclear magnetic properties - the spin quantum number, I, the natural abundance, $\mathrm{N}$, the Larmor frequency (at $14.1 \mathrm{~T}$ ), $\omega_{0}$, relative receptivity, and (for I $>1 / 2$ ) nuclear electric quadrupole moment, $\mathrm{Q}$ - of some nuclei commonly found in microporous materials.

\begin{tabular}{|c|c|c|c|c|c|c|}
\hline Nucleus & I & $\mathrm{N}(\%)$ & $\omega_{0} / \mathrm{MHz}$ & Receptivity $^{a}$ & $\mathrm{Q} / \mathrm{fm}^{2}$ & $\begin{array}{c}\text { Suitable for } \\
\text { routine study? }\end{array}$ \\
\hline${ }^{1} \mathrm{H}$ & $1 / 2$ & 99.99 & 600.13 & 1.00 & & yes \\
\hline${ }^{2} \mathrm{H}$ & 1 & 0.01 & 92.12 & $1.11 \times 10^{-6}$ & 0.268 & \\
\hline${ }^{13} \mathrm{C}$ & $1 / 2$ & 1.07 & 150.90 & $1.70 \times 10^{-4}$ & & yes \\
\hline${ }^{14} \mathrm{~N}$ & 1 & 99.64 & 43.37 & $1.00 \times 10^{-3}$ & 2.044 & \\
\hline${ }^{15} \mathrm{~N}$ & $1 / 2$ & 0.36 & 60.83 & $3.79 \times 10^{-6}$ & & \\
\hline${ }^{17} \mathrm{O}$ & $5 / 2$ & 0.04 & 81.36 & $1.10 \times 10^{-5}$ & -2.558 & \\
\hline${ }^{19} \mathrm{~F}$ & $1 / 2$ & 100.00 & 564.69 & $8.33 \times 10^{-1}$ & & yes \\
\hline${ }^{23} \mathrm{Na}$ & $3 / 2$ & 100.00 & 158.75 & $9.26 \times 10^{-2}$ & 10.4 & yes \\
\hline${ }^{25} \mathrm{Mg}$ & $5 / 2$ & 10.00 & 36.74 & $2.68 \times 10^{-4}$ & 19.94 & \\
\hline${ }^{27} \mathrm{Al}$ & $5 / 2$ & 100.00 & 156.38 & $2.06 \times 10^{-1}$ & 14.66 & yes \\
\hline${ }^{29} \mathrm{Si}$ & $1 / 2$ & 4.69 & 119.23 & $3.67 \times 10^{-4}$ & & yes \\
\hline${ }^{31} \mathrm{P}$ & $1 / 2$ & 100.00 & 242.94 & $6.63 \times 10^{-2}$ & & yes \\
\hline${ }^{45} \mathrm{Sc}$ & $7 / 2$ & 100.00 & 145.78 & $3.01 \times 10^{-1}$ & -22.0 & yes \\
\hline${ }^{67} \mathrm{Zn}$ & $5 / 2$ & 4.10 & 37.55 & $1.17 \times 10^{-4}$ & 15.0 & \\
\hline${ }^{69} \mathrm{Ga}$ & $3 / 2$ & 60.11 & 144.04 & $4.16 \times 10^{-2}$ & 17.1 & \\
\hline${ }^{71} \mathrm{Ga}$ & $3 / 2$ & 39.89 & 183.02 & $5.66 \times 10^{-2}$ & 10.7 & \\
\hline${ }^{87} \mathrm{Rb}$ & $3 / 2$ & 27.83 & 196.37 & $4.88 \times 10^{-2}$ & 13.35 & yes \\
\hline${ }^{91} \mathrm{Zr}$ & $5 / 2$ & 11.22 & 55.79 & $1.05 \times 10^{-3}$ & -17.6 & \\
\hline
\end{tabular}

a Receptivity is defined as $\gamma^{3} \mathrm{~N}(\mathrm{I}(\mathrm{I}+1))$, where $\gamma$ is the gyromagnetic ratio of the nucleus, and quoted relative to ${ }^{1} \mathrm{H}$. 


\section{References}

1. P. A. Wright, Microporous Framework Solids, $1^{\text {st }}$ edn., The Royal Society of Chemistry, 2008.

2. M. Mastalerz, Chem. Eur. J., 2012, 18, 10082.

3. G. Férey, Chem. Soc. Rev., 2008, 37, 191.

4. L. Mafra and J. Klinowski, Molecular Sieves: Crystalline Systems, eMagRes, 2013. DOI: 10.1002/9780470034590.emrstm1325.

5. L. Mafra and J. Klinowski, Advanced Solid State NMR Techniques for the Study of Molecular Sieves, eMagRes, 2013. DOI: 10.1002/9780470034590.emrstm1307.

6. A. Sutrisno and Y. Huang, Solid State Nucl. Magn. Reson., 2013, 49, 1.

7. Y. Jiang, J. Huang, W. Dai and M. Hunger, Solid State Nucl. Magn. Reson., 2011, 39, 116.

8. A. Zheng, S.-J. Huang, Q. Wang, H. Zhang, F. Deng and S.-B Liu, Chinese J. Catal., 2013, 34, 436 .

9. C. Bonhomme, C. Gervais, F. Babonneau, C. Coelho, F. Pourpoint, T. Azaïs, S. E. Ashbrook, J. M. Griffin, J. R. Yates, F. Mauri and C. J. Pickard, Chem. Rev., 2012, 112, 5733.

10. C. Bonhomme, C. Gervais and D. Laurencin, Prog. Nucl. Magn. Reson. Spectrosc., 2014, 77, 1 .

11. C. Baerlocher and L. B. McCusker, Database of Zeolite Structures, http://www.izastructure.org/databases, Accessed $5^{\text {th }}$ Feb, 2014.

12. L. Pauling, Z. Kristallogr., 1930, 74, 213.

13. S. T. Wilson, B. M. Lok, C. A. Messina, T. R. Cannan and E. M. Flanigen, J. Am. Chem. Soc., 1982, 104, 1146.

14. A. Phan, C. J. Doonan, F. J. Uribe-Romo, C. B. Knobler, M. O'Keeffe and O. M. Yaghi, Acc. Chem. Res., 2010, 43, 58.

15. R. E. Morris and S. J. Weigel, Chem. Soc. Rev., 1997, 26, 309.

16. E. R. Parnham and R. E. Morris, Acc. Chem. Res., 2007, 40, 1005.

17. R. E. Morris and S. L. James, Angew. Chem. Int. Ed., 2013, 52, 2163.

18. E. R. Parnham, PhD Thesis, University of St Andrews, 2006.

19. L. Han, Y. Wang, S. Zhang and X. Lu, J. Crystal Growth, 2008, 311, 167.

20. S. Girard, J. D. Gale, C. Mellot-Draznieks and G. Férey, J. Am. Chem. Soc., 2002, 124, 1040.

21. M. Amri, S. E. Ashbrook, D. M. Dawson, J. M. Griffin, R. I. Walton and S. Wimperis, J. Phys. Chem. C, 2012, 116, 15048.

22. M. Richter, H. L. Zubowa, R. Eckelt and R. Fricke, Micropor. Mater., 1996, 7, 119.

23. K. K. Tanabe and S. M. Cohen, Chem. Soc. Rev., 2011, 40, 498.

24. M. I. H. Mohideen, B. Xiao, P. S. Wheatley, A. C. McKinlay, Y. Li, A. M. Z. Slawin, D. W. Aldous, N. F. Cessford, T. Dueren, X. Zhao, R. Gill, K. M. Thomas, J. M. Griffin, S. E. 
Ashbrook and R. E. Morris, Nature Chem., 2011, 3, 304.

25. All articles in the special edition of Chem. Soc. Rev. 2009, 38.

26. NMR Crystallography, Eds R. K. Harris, R. E. Wasylishen and M. J. Duer, Wiley 2009.

27. T. Charpentier, Solid State Nucl. Magn. Reson., 2011, 40, 1.

28. J. Cuny, S. Messaoudi, V. Alonzo, E. Furet, J.-F. Halet, E. Le Fur, S. E. Ashbrook, C. J. Pickard, R. Gautier and L. Le Polles, J. Comp. Chem., 2008, 29, 2279.

29. D. C. Apperley, R. K. Harris and P. Hodgkinson, Solid-State NMR: Basic Principles and Practice, Momentum Press, 2012.

30. J. P. S. Mowat, S. R. Miller, J. M. Griffin, V. R. Seymour, S. E. Ashbrook, S. P. Thompson, D. Fairen-Jimenez, A. M. Banu, T. Duren and P. A. Wright, Inorg. Chem., 2011, $50,10844$.

31. V. R. Seymour, E. C. V. Eschenroeder, M. Castro, P. A. Wright and S. E. Ashbrook, Cryst. Eng. Comm., 2013, 15, 8668.

32. NMR of Quadrupolar Nuclei in Solid Materials, Eds. R. E. Wasylishen, S. E. Ashbrook and S. Wimperis, Wiley, 2012.

33. S. E. Ashbrook, M. Cutajar, J. M. Griffin, Z. A. D. Lethbridge, R. I. Walton and S. Wimperis, J. Phys. Chem. C, 2009, 113, 10780.

34. J. P. S. Mowat, V. R. Seymour, J. M. Griffin, S. P. Thompson, A. M. Z Slawin, D. Fairen-Jimenez, T. Duren, S. E. Ashbrook and P. A. Wright, Dalton Transact., 2012, 41, 3937. 35. J. P. S. Mowat, S. R. Miller, A. M. Z. Slawin, V. R. Seymour, S. E. Ashbrook and P. A. Wright, Micropor. Mesopor. Mater., 2011, 142, 322.

36. R. Dupree, Double Rotation NMR, eMagRes, 2011. DOI: 10.1002/9780470034590.emrstm1203.

37. Y. Wu, B. F. Chmelka, A. Pines, M. E. Davis, P. J. Grobet and P. A. Jacobs, Nature, 1990, 346, 550 .

38. F. Taulelle, A. Samoson, T. Loiseau and G. Ferey, J. Phys. Chem. B, 1998, 102, 8588.

39. G. Engelhardt, H. Keller, P. Sieger, W. Depmeier and A. Samoson, Solid State Nucl. Magn. Reson., 1992, 1, 127.

40. M. Kovalakova and P. J. Grobet, Appl. Magn. Reson., 1996, 10, 447.

41. L. M. Bull, B. Bussemer, T. Anupold, A. Reinhold, A. Samoson, J. Sauer, A. K. Cheetham and R. Dupree, J. Am. Chem. Soc., 2000, 122, 4948.

42. J. M. Griffin, L. Clark, V. R. Seymour, D. W. Aldous, D. M. Dawson, D. Iuga, R. E. Morris and S. E. Ashbrook, Chem. Sci., 2012, 3, 2293.

43. L. Frydman and J. S. Harwood, J. Am. Chem. Soc., 1995, 117, 5367.

44. Z. Gan, J. Am. Chem. Soc., 2000, 122, 3242.

45. S. E. Ashbrook and S. Wimperis, Prog. Nucl. Magn. Reson. Spec., 2004, 45, 53.

46. S. Antonijevic, S. E. Ashbrook, S. Biedasek, R. I. Walton, S. Wimperis and H. X. Yang, J. Am Chem. Soc., 2006, 128, 8054.

47. T. Gullion and J. Schaefer, J. Magn. Reson., 1989, 81, 196. 
48. C. P. Grey and A. J. Vega, J. Am. Chem. Soc., 1995, 117, 8232.

49. A. Abraham, R. Prins, J. A. van Bokhoven, E. R. H. van Eck and A. P. M. Kentgens, Solid State Nucl. Magn. Reson., 2009, 35, 61.

50. H. Huo, L. M. Peng, Z. H. Ga and C. P. Grey, J. Am. Chem. Soc., 2012, 134, 9708.

51. X. Kong, H. Deng, F. Yan, J. Kim, J. A. Swisher, B. Smit, O. M. Yaghi, and J. A. Reimer, Science, 2013, 341, 882.

52. C. A. Fyfe, H. Meyer zu Altenschildesche, K. C. Wong-Moon, H. Grondey and J. M. Chezeau, Solid State Nucl. Magn. Reson., 1997, 9, 97.

53. C. Martineau, B. Bouchevreau, T. Zhijian, S. J. Lohmeier, P. Behrens and F. Taulelle, Chem. Mater., 2011, 23, 4799

54. G. Poulet, A. Tuel and P. Sautet, J. Phys. Chem. B, 2005, 109, 22939.

55. G. Mali, V. Kaucic and F. Taulelle, J. Chem. Phys., 2008, 128, 204503.

56. C. Fernandez, C. Morais, J. Rocha and M. Pruski, Solid State Nucl. Magn. Reson., $2002,21,61$.

57. J. W. Wiench, G. Tricot, L. Delevoye, J. Trebosc, J. Frye, L. Montague, J. P. Amoureux and M. Pruski, Phys. Chem. Chem. Phys., 2006, 8, 144.

58. C. M. Morais, V. Montouillout, M. Deschamps, D. Iuga, F. Fayon, F. A. A. Paz, J. Rocha, C. Fernandez and D. Massiot, Magn. Reson. Chem., 2009, 47, 942.

59. A. Sutrisno, V. V. Terskikh, Q. Shi, Z. Song, J. Dong, S. Y. Ding, W. Wang, B. R. Provost, T. D. Daff, T. K. Woo and Y. Huang, Chem. Eur. J., 2012, 18, 12251.

60. J. Xu, V. V. Terskikh and Y. Huang, J. Phys. Chem. Lett., 2013, 4, 7.

61. J. Zhu, Z. Lin, Z. Yan and Y. Huang, Chem. Phys. Lett., 2008, 461, 260.

62. E. Kupce and R. Freeman, J. Magn. Reson. A, 1995, 115, 273.

63. H. Y. Carr and E. M. Purcell, Phys. Rev., 1954, 94, 630.

64. S. Meiboom and D. Gill, Rev. Sci. Instrum., 1958, 29, 688.

65. S. Devautour-Vinot, G. Maurin, C. Serre, P. Horcajada, D. Paula da Cunha, V. Guillerm, E. de Souza Costa, F. Taulelle and C. Martineau, Chem. Mater., 2012, 24, 2168.

66. C. J. Pickard and F. Mauri, Phys. Rev. B, 2001, 63, 245101.

67. S. E. Ashbrook, M. Cutajar, C. J. Pickard, R. I. Walton and S. Wimperis, Phys. Chem. Chem. Phys., 2008, 10, 5754.

68. M. Profeta, C. J. Pickard and F. Mauri, J. Am. Chem. Soc., 2003, 125, 541.

69. S. E. Ashbrook and D. M. Dawson, Acc. Chem. Res. 2013, 46, 1964.

70. S. Sneddon, D. M. Dawson, C. J. Pickard and S. E. Ashbrook, Phys. Chem. Chem. Phys., 2014, 16, 2660.

71. P. J. Byrne, J. E. Warren, R. E. Morris and S. E. Ashbrook, Solid State Sci., 2009, 11, 1001.

72. B. K. Chang, N. C. Bristowe, P. D. Bristowe and A. K. Cheetham, Phys. Chem. Chem. Phys., 2012, 14, 7059.

73. A. M. Walker, B. Civalleri, B. Slater, C. Mellot-Draznieks, F. Corà, C. Zircovich- 
Wilson, G. Román-Pérez, J. M. Soler and J. D. Gale, Angew. Chem. Int. Ed., 2010, 49, 7501.

74. E. R. McNellis, J. Meyer and K. Reuter, Phys. Rev. B, 2009, 80, 205414.

75. C. Martineau, A. Cadiau, B. Bouchevreau, J. Senker, F. Taulelle and K. Adil, Dalton Transact., 2012, 41, 6232 .

76. P. J. Barrie and J. Klinowski, J. Phys. Chem., 1989, 93, 5972.

77. C. Martineau, B. Bouchevreau, Z. Tian, S.-J. Lohmeier, P. Behrens and F. Taulelle, Chem. Mater., 2011, 23, 4799.

78. C. Martineau, C. Mellot-Draznieks and F. Taulelle, Phys. Chem. Chem. Phys., 2011, $13,18078$.

79. D. H. Brouwer, J. Am. Chem. Soc., 2008, 130, 6306.

80. D. H. Brouwer, Solid State Nucl. Magn. Reson., 2013, 51, 37.

81. D. H. Brouwer, R. J. Darton, R. E. Morris and M. H. Levitt, J. Am. Chem. Soc., 2005, $127,10365$.

82. D. H. Brouwer, I. L. Moudrakovski, R. J. Darton and R. E. Morris, Magn. Reson. Chem., 2010, 48, S113.

83. R. L. Vold and G. L. Hoatson, J. Magn. Reson., 2009, 198, 57.

84. J. Gonzalez, R. N. Devi, D. P. Tunstall, P. A. Cox and P. A. Wright, Micropor. Mesopor. Mater., 2005, 84, 97.

85. D. I. Kolokolov, H. Jobic, A. G. Stepanov, V. Guillerm, T. Devic, C. Serre and G. Férey, Angew. Chem. Int. Ed., 2010, 49, 4791.

86. F. Gul-E-Noor, D. Michel, H. Krautscheid, J. Haase and M. Bertmer, Micropor. Mesopor. Mater., 2013, 180, 8.

87. J. F. Stebbins, P. D. Zhao, S. K. Lee and X. Cheng, Am. Mineral., 1999, 84, 1680.

88. M. Müller, S. Hermes, K. Kähler, M. W. E. van den Berg, M. Muhler and R. A. Fischer, Chem. Mater., 2008, 20, 4576.

89. P. He, J. Xu, V. V. Terskikh, A. Sutrisno, H.-Y. Nie and Y. Huang, J. Phys. Chem. C, 2013, 117, 16953.

90. L. M. Bull, A. K. Cheetham, T. Anupõld, A. Reinhold, A. Samoson, J. Sauer, B. Bussemer, Y. Lee, S. Gann, J. Shore, A. Pines and R. Dupree, J. Am. Chem. Soc., 1998, 120, 3510.

91. J. F. Haw, Phys. Chem. Chem. Phys., 2002, 4, 5431.

92. D. M. Dawson, L. E. Jamieson, M. I. H. Mohideen, A. C. McKinlay, I. A. Smellie, R. Cadou, N. S. Keddie, R. E. Morris and S. E. Ashbrook, Phys. Chem. Chem. Phys., 2013, 15, 919.

93. A. G. Stepanov, Russ. Chem. Rev., 1999, 68, 563.

94. D. Farcasiu, R. Leu and A. Corma, J. Phys. Chem. B, 2002, 106, 928.

95. G. P. Holland, B. R. Cherry and T. M. Alam, J. Phys. Chem. B, 2004, 108, 16420.

96. W. Morris, R. E. Taylor, C. Dybowski, O. M. Yaghi and M. A. Garcia-Garibay, J. Mol. Struct., 2011, 1004, 94. 
97. C. A. Fyfe, H. Gies and Y. Feng, J. Am. Chem. Soc., 1989, 111, 7702.

98. M. Haouas and F. Taulelle, J. Phys. Chem. B, 2006, 110, 3007.

99. P. J. Barrie and J. Klinowski, Prog. Nucl. Magn. Reson. Spectrosc. 1992, $24,91$.

100. I. L. Moudrakovski, A. Nossov, S. Lang, S. R. Breeze, C. I. Ratcliffe, B. Simard, G. Santyr and J. A. Ripmeester, Chem. Mater., 2000, 12, 1181.

101. K. J. Ooms and R. E. Wasylishen, Micropor. Mesopor. Mater., 2007, 103, 341.

102. A. Tuel, S. Caldarelli, A. Meden, L. B. McCusker, C. Baerlocher, A. Ristic, N. Rajic, G. Mali and V. Kaucic, J. Phys. Chem. B, 2000, 104, 5697.

103. X. Kong, E. Scott, W. Ding, J. A. Mason, J. R. Long and J. A. Reimer, J. Am. Chem. Soc., 2012, 134, 14341.

104. T. Loiseau, C. Serre, C. Huguenard, G. Fink, F. Taulelle, M. Henry, T. Bataille and G. Férey, Chem. Eur. J., 2004, 10, 1373.

105. P. Horcajada, C. Serre, G. Maurin, N. A. Ramsahye, F. Balas, M. Vallet-Regi, M. Sebban, F. Taulelle and G. Férey, J. Am. Chem. Soc., 2008, 130, 6774.

106. Z. T. Lalowicz, G. Stoch, A. Birczynski, M. Punkkinen, E. E. Ylinen, M. Krzystyniak, K. Gora-Marek and J. Datka, Solid State Nucl. Magn. Reson., 2012, 45-46, 66.

107. T. Ueda, K. Kurokawa, H. Omichi, K. Miyakubo and T. Eguchi, Chem. Phys. Lett., 2007, 443, 293.

108. S. H. Li, A. M. Zheng, Y. C. Su, H. L. Zhang, L. Chen, J. Yang, C. H. Ye and F. Deng, J. Am. Chem. Soc., 2007, 129, 11161.

109. M. C. Xu, A. Arnold, A. Buchholz, W. Wang and M. Hunger, J. Phys. Chem. B, 2002, $106,12140$.

110. J. F. Haw, J. B. Nicholas, T. Xu, L. W. Beck and D. B. Ferguson, Acc. Chem. Res., 1996, $29,259$.

111. W. Dai, X. Wang, G. Wu, L. Li, N. Guan and M. Hunger, Chem. Cat. Chem., 2012, 4, 1428.

112. V. I. Bakhmutov, Solid-State NMR in Materials Science, CRC Press, 2012.

113. G. Mali, A. Ristic and V. Kaucic, J. Phys. Chem. B 2005, 109, 10711.

114. G. Kervern, S. Steuernagel, F. Engelke, G. Pintacuda and L. Emsley, J. Am. Chem. Soc., 2007, 129, 14118.

115. P. Horcajada, C. Serre, M. Vallet-Regi, M. Sebban, F. Taulelle and G. Férey, Angew. Chem. Int. Ed., 2006, 45, 5974.

116. G. W. Peterson, G. W. Wagner, A. Balboa, J. Mahle, T. Sewell and C. J. Karwacki, J. Phys. Chem. C, 2009, 113, 13906.

117. F. Gul-E-Noor, B. Jee, A. Pöppl, M. Hartmann, D. Himsl and M. Bertmer, Phys. Chem. Chem. Phys., 2011, 13, 7783.

118. G. de Combarieu, M. Morcrette, F. Millange, N. Guillou, J. Cabana, C. P. Grey, I. Margiolaki, G. Férey and J. M. Tarascon, Chem. Mater., 2009, 21, 1602.

119. R. A. Wind, Dynamic Nuclear Polarization and High-Resolution NMR of Solids, 
eMagRes, 2007. DOI: 10.1002/9780470034590.emrstm0140.

120. F. Blanc, S. Y. Chong, T. O. McDonald, D. J. Adams, S. Pawsey, M. A. Caporini and A. I. Cooper, J. Am. Chem. Soc., 2013, 135, 15290.

121. F. Pourpoint, A. S. L. Thankamony, C. Volkringer, T. Loiseau, J. Trébosc, F. Aussenac, D. Carnevale, G. Bodenhausen, H. Vezin, O. Lafon and J. P. Amoureux, Chem. Commun., 2014, 50, 933.

122. M. Concistrè, O. G. Johannessen, E. Carignani, M. Geppi and M. H. Levitt, Acc. Chem. Res., 2013, 46, 1914.

123. L. van Wüllen, G. Schwering, E. Naumann and M. Jansen, Solid State Nucle. Magn. Reson., 2004, 26, 84 .

124. A. Vjunov, M. Y. Hu, J. Feng, D. M. Camaioni, D. Mei, J. Z. Hu, C. Zhao, and J. A. Lercher, Angew. Chemie, 2014, 53, 479.

125. M. Hunger, Prog. Nucl. Magn. Reson. Spectrosc., 2008, 53, 105.

126. I. I. Ivanova and Y. G. Kolyagin, Chem. Soc. Rev. 2010, 39, 5018.

127. J. Z. Hu, J. A. Sears, H. S. Mehta, J. J. Ford, J. H. Kwak, K. Zhu, Y. Wang, J. Liu, D. W. Hoyt and C. H. F. Peden, Phys. Chem. Chem. Phys., 2012, 14, 2137.

128. S. T. Roberts, M. P. Renshaw, M. Lutecki, J. McGregor, A. J. Sederman, M. D. Mantle and L. F. Gladden, Chem. Commun., 2013, 49, 10519. 


\section{Figure Captions}

Figure 1. (a) The sodalite (SOD) framework type, ${ }^{11}$ with vertices and links indicated. Some example vertices and links are (b) $\mathrm{SiO}_{4}$ and $\mathrm{AlO}_{4}$ tetrahedra, connected by Si-O-Si and SiO-Al linkages in the aluminosilicate zeolite, sodalite, ${ }^{12}$ (c) $\mathrm{AlO}_{4}$ and $\mathrm{PO}_{4}$ tetrahedra connected by Al-O-P linkages in the aluminophosphate, AlPO-20, ${ }^{13}$ and (d) $\mathrm{Zn}^{2+}$ connected by imidazolate linkers in the zeolitic imidazolate framework, ZIF-8. ${ }^{14}$ Modification of the framework may occur through (e) post-synthetic modification, direct synthesis including the modified linkers/vertices, or in situ modification of linker/vertex precursors.

Figure 2. (a) ${ }^{13} \mathrm{C}$ MAS NMR spectra of $\mathrm{Sc}_{2} \mathrm{BDC}_{3}$ and $\mathrm{Sc}_{2}\left(\mathrm{NH}_{2}-\mathrm{BDC}\right)_{3}^{30}$ and structures of the organic linkers molecules used, (b) ${ }^{27}$ Al MAS NMR spectra of JDF-2 and AlPO-53(B) ${ }^{33}$ and (c) ${ }^{45}$ Sc MAS and MQMAS spectra, and corresponding crystal structures, of dried and hydrated MIL-53(Sc). ${ }^{34}$ Spectra are (a) reprinted (adapted) with permission from Ref. 30. Copyright (2011) American Chemical Society, (b) reprinted (adapted) with permission from Ref. 33. Copyright (2009) American Chemical Society, and (c) reproduced (from Ref. 34) by permission of The Royal Society of Chemistry.

Figure 3. (a) ${ }^{27} \mathrm{Al}$ and ${ }^{31} \mathrm{P}$ MAS spectra of as-made AlPO STA-2 and (b) ${ }^{27} \mathrm{Al} /{ }^{31} \mathrm{P}$ HETCOR spectrum of AlPO STA-2 and plots of calculated shifts for models. ${ }^{31}$ Figures are adapted with permission from Ref. 31 - Published by the Royal Society of Chemistry.

Figure 4. (a) Overview of the structure determination strategy combining solid-state NMR spectroscopy and X-ray diffraction. ${ }^{81}$ (b) ${ }^{29} \mathrm{Si}$ MAS and two-dimensional homonuclear double-quantum correlation NMR spectra, and (c) corresponding plots of the build up of cross peak signal intensity, used to unambiguously determine the structure of an unknown high-silica zeolite (later identified as ITQ-4) in a blind test. ${ }^{81}$ (d) Overlay of the crystal structure of ITQ-4, determined by NMR-crystallography and X-ray crystallography 
alone. Adapted with permission from Ref. 81. Copyright (2005) American Chemical Society.

Figure 5. (a) Variable-temperature experimental ${ }^{2} \mathrm{H}(9.4 \mathrm{~T})$ wide-line NMR spectra of fullydeuterated $\mathrm{Sc}_{2} \mathrm{BDC}_{3}{ }^{30}$ Reprinted (adapted) with permission from Ref. 30. Copyright (2011) American Chemical Society. ${ }^{17} \mathrm{O}(20.0 \mathrm{~T})$ (b) DOR and (c) MQMAS spectra of as-made and calcined $\mathrm{AlPO}_{4}$ SIZ-4, enriched in ${ }^{17} \mathrm{O}$ using an ionothermal synthesis approach. ${ }^{42}$ Isotropic resonances are highlighted by dots. Reproduced from Ref. 42 with permission from the Royal Society of Chemistry.

Figure 6. (a) ${ }^{129} \mathrm{Xe}$ NMR spectra of hyperpolarised ${ }^{129} \mathrm{Xe}$ in IRMOF-8 as a function of temperature. $^{101}$ Reprinted from Ref. 101, Copyright (2007), with permission from Elsevier. (b) ${ }^{15} \mathrm{~N}$ CP MAS NMR of zeolite $3 \mathrm{~A}$ as a function of ammonia loading and the cation site occupied by $\mathrm{Na}^{+}$in the six-membered ring. ${ }^{95}$ The resonances arising from the two distinct adsorbed ammonia species, I and II, are indicated in the spectrum obtained at a loading of $1.66 \mathrm{mmol} \mathrm{g}^{-1}$. Adapted with permission from Ref. 95. Copyright (2004) American Chemical Society. (c) ${ }^{13} \mathrm{C}$ CSA powder patterns of ${ }^{13} \mathrm{C}$-enriched $\mathrm{CO}_{2}$ in $\mathrm{Mg} \mathrm{CPO}-$ 27 at various temperatures. ${ }^{103}$ Adapted with permission from Ref. 103. Copyright (2012) American Chemical Society. (d) ${ }^{27} \mathrm{Al}$ (14.1 T) MQMAS and STMAS NMR spectra of asmade AlPO-14 prepared with an isopropylammonium template. After Antonijevic et al. $(2006){ }^{46}$

Figure 7. (a) Schematic representation of the spin-echo mapping technique used for recording broad phosphorus NMR spectra and (b) wideline ${ }^{31} \mathrm{P}$ NMR in several as-made and calcined rehydrated MeAlPO4-34. ${ }^{113}$ Adapted with permission from Ref. 113. Copyright (2005) American Chemical Society. (c, d) ${ }^{13} \mathrm{C}(14.1 \mathrm{~T}, 60 \mathrm{kHz}$ MAS) NMR spectra of the Cu-based MOFs, HKUST-1 and STAM-1, synthesised with (c) natural-abundance linkers, (d) linkers with ${ }^{13} \mathrm{C}$-labelled $\mathrm{CO}_{2}$ species and (e) linkers with ${ }^{13} \mathrm{C}$ - labelled benzene 
rings. The structure of the linkers is shown, with isotopically-enriched C species highlighted in green. After Dawson et al. Ref. 92. 


\section{Graphical Abstract}

This Perspective describes how solid-state NMR spectroscopy can be used to investigate the structure, disorder, dynamics and reactivity of crystalline microporous framework materials. 

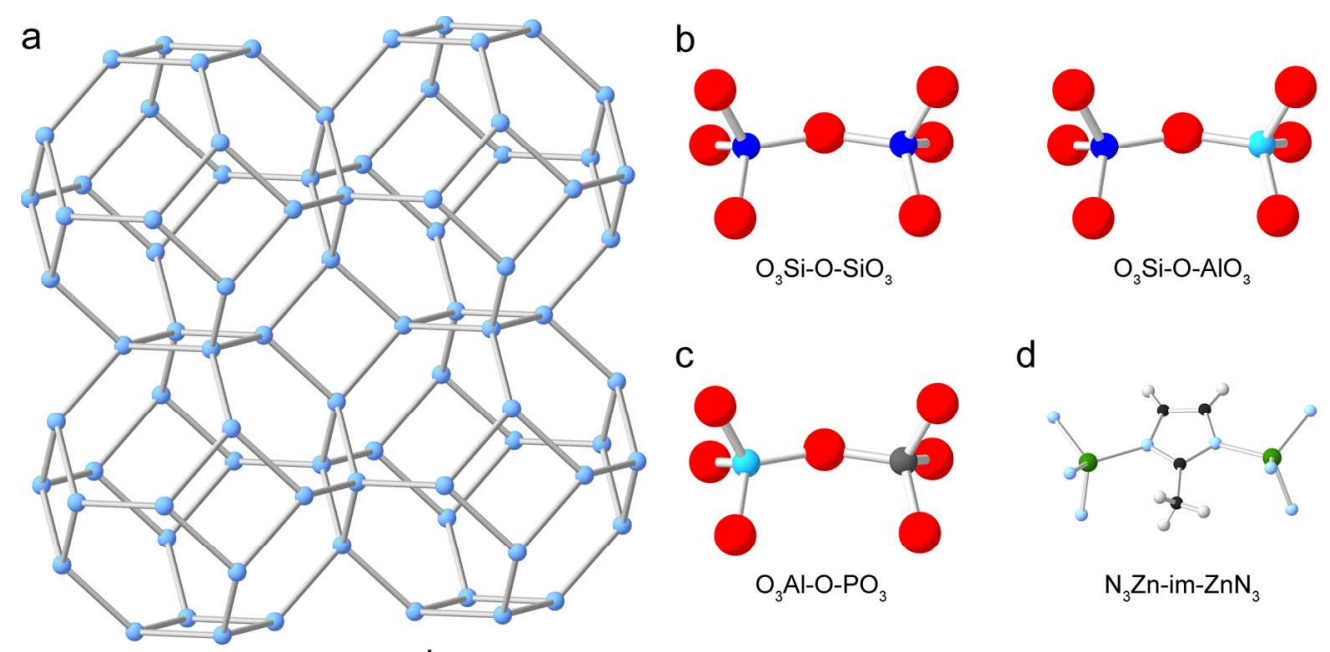

e
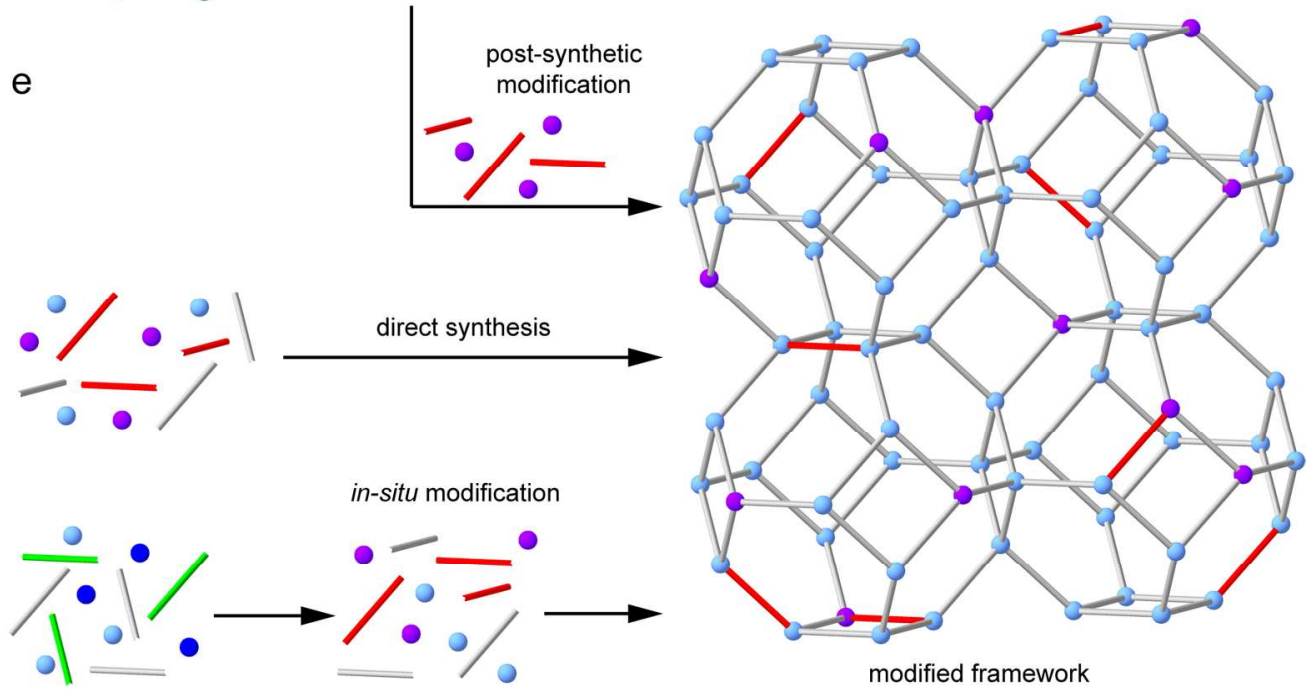

in-situ modification

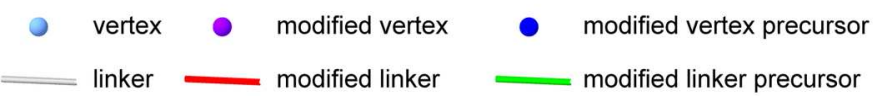

$162 \times 181 \mathrm{~mm}(300 \times 300 \mathrm{DPI})$ 

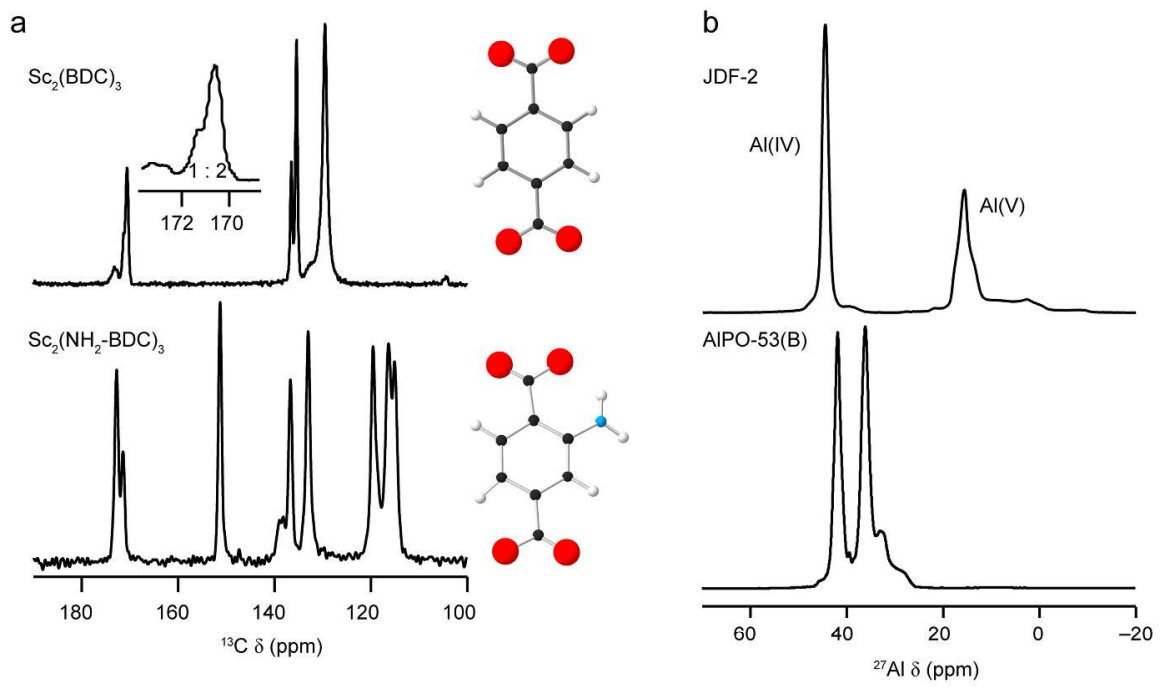

c
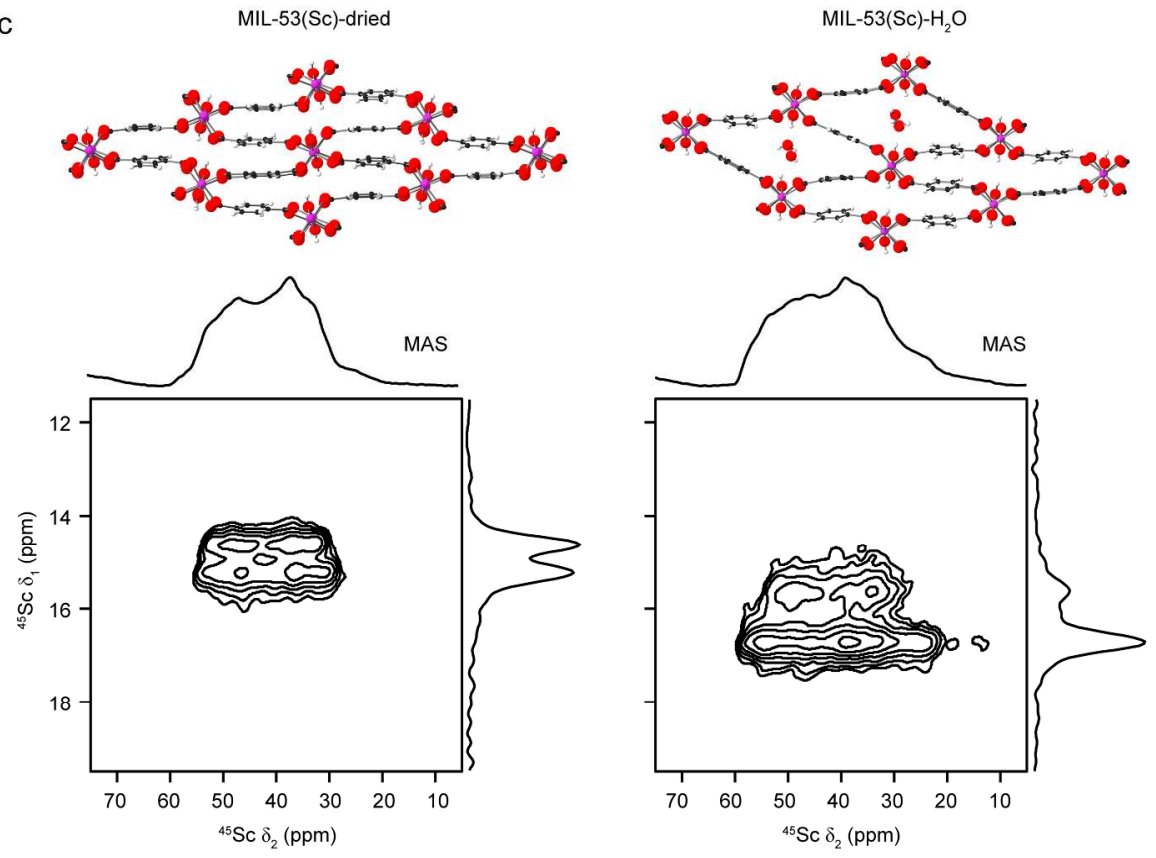

$215 \times 293 \mathrm{~mm}(300 \times 300$ DPI $)$ 
a
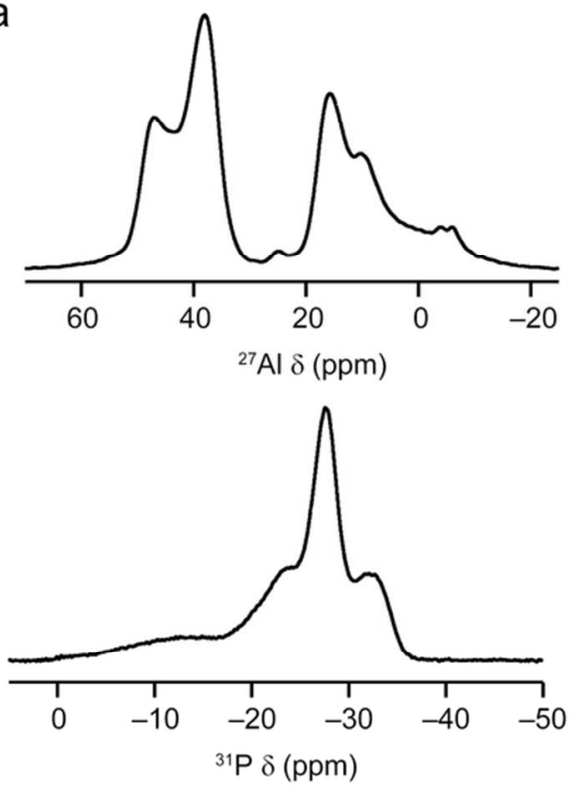

b

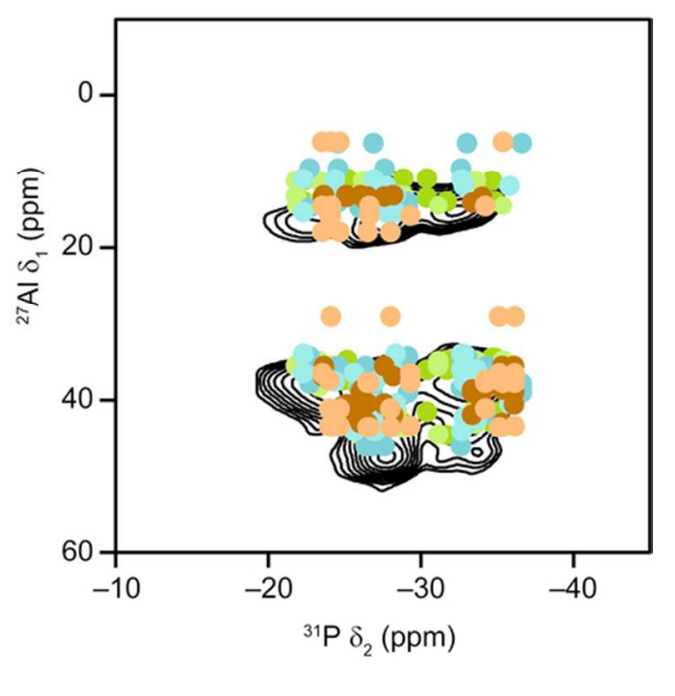

$78 \times 47 \mathrm{~mm}(300 \times 300 \mathrm{DPI})$ 
a

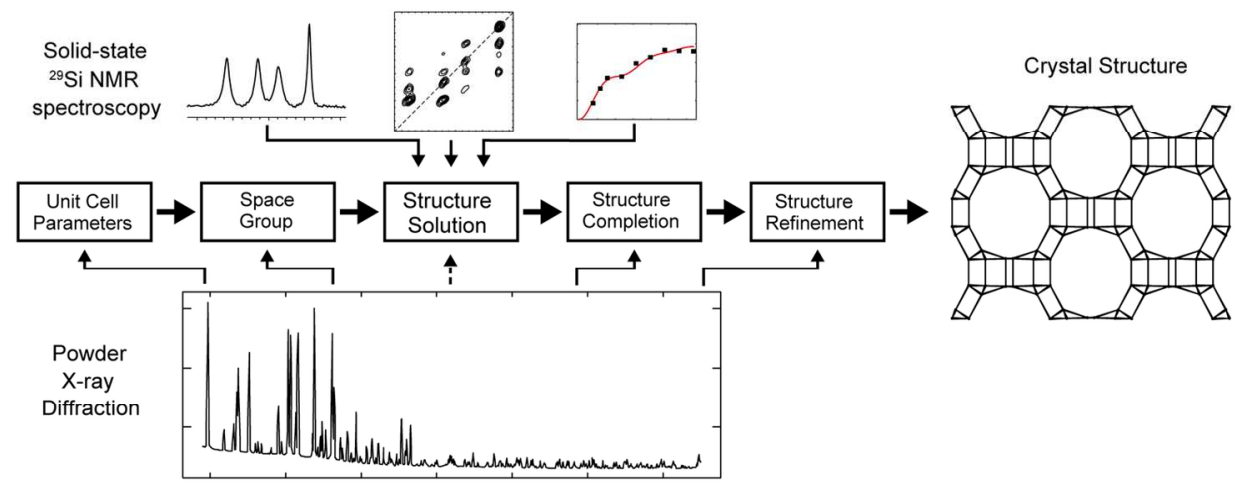

b

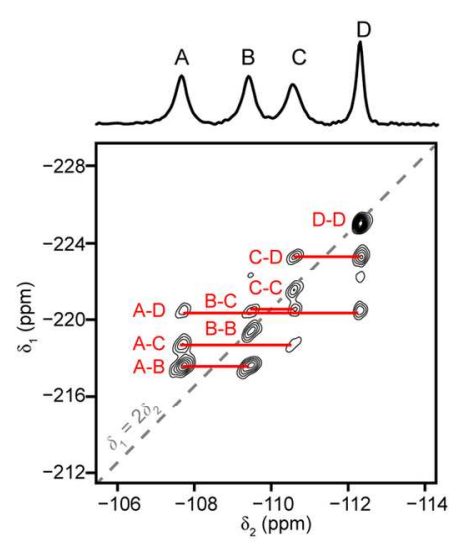

c

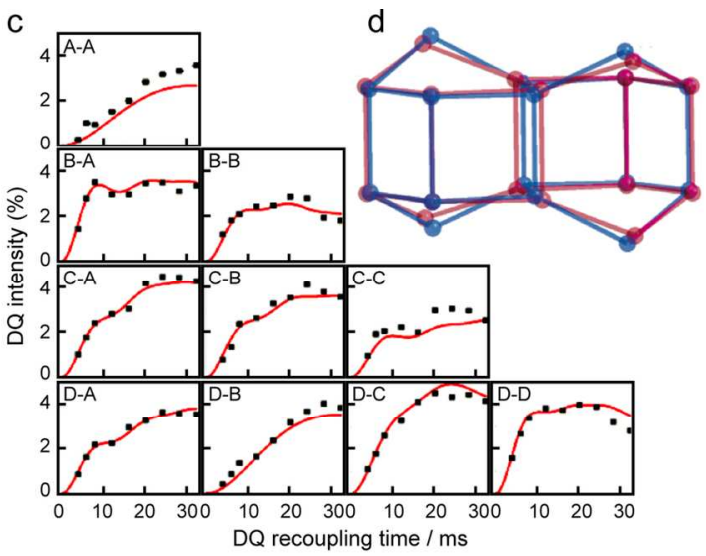

$140 \times 117 \mathrm{~mm}(300 \times 300$ DPI $)$ 

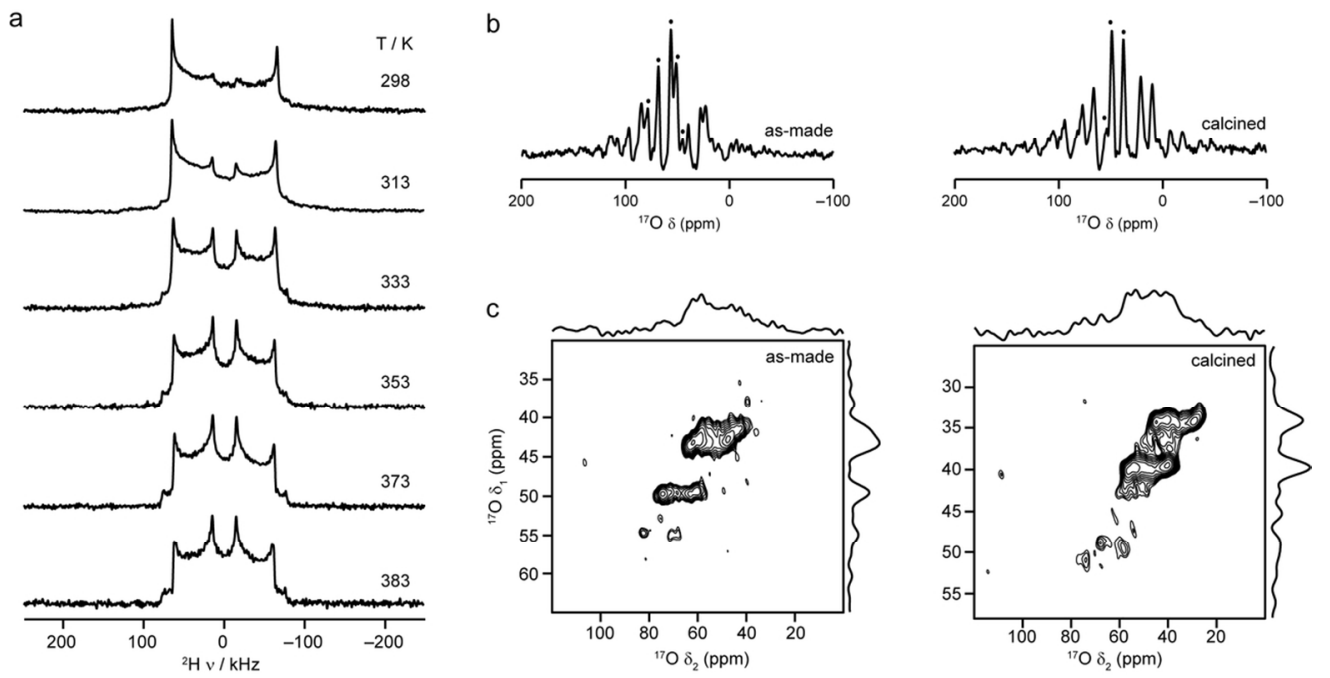

$107 \times 55 \mathrm{~mm}(300 \times 300$ DPI $)$ 
a

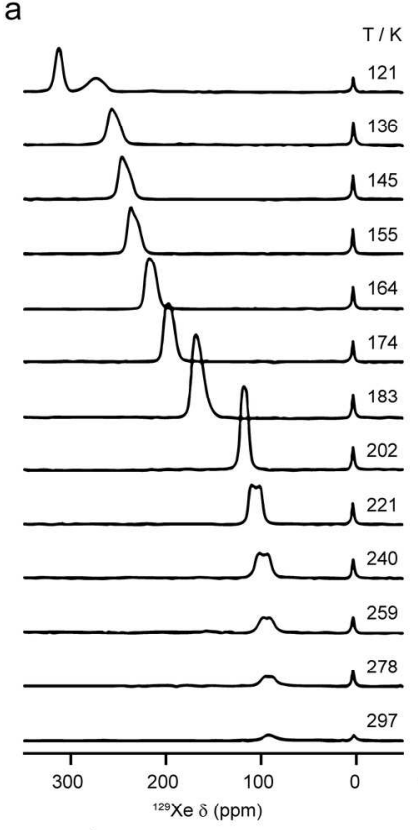

d h n

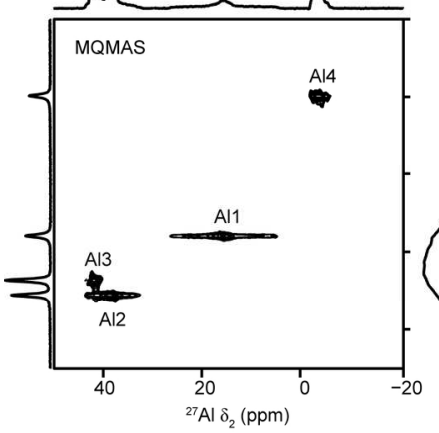

b
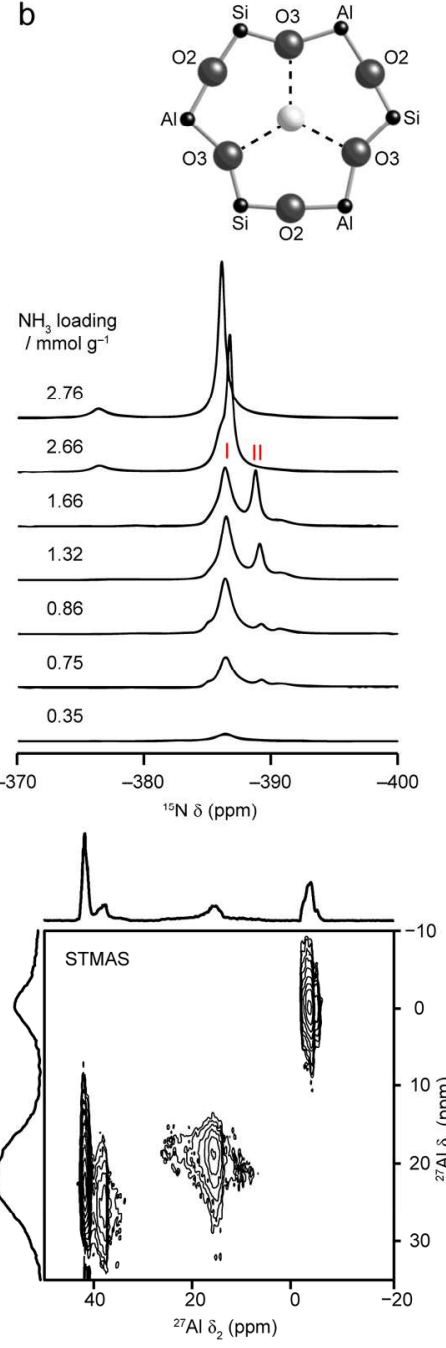

$177 \times 185 \mathrm{~mm}(300 \times 300$ DPI $)$
C
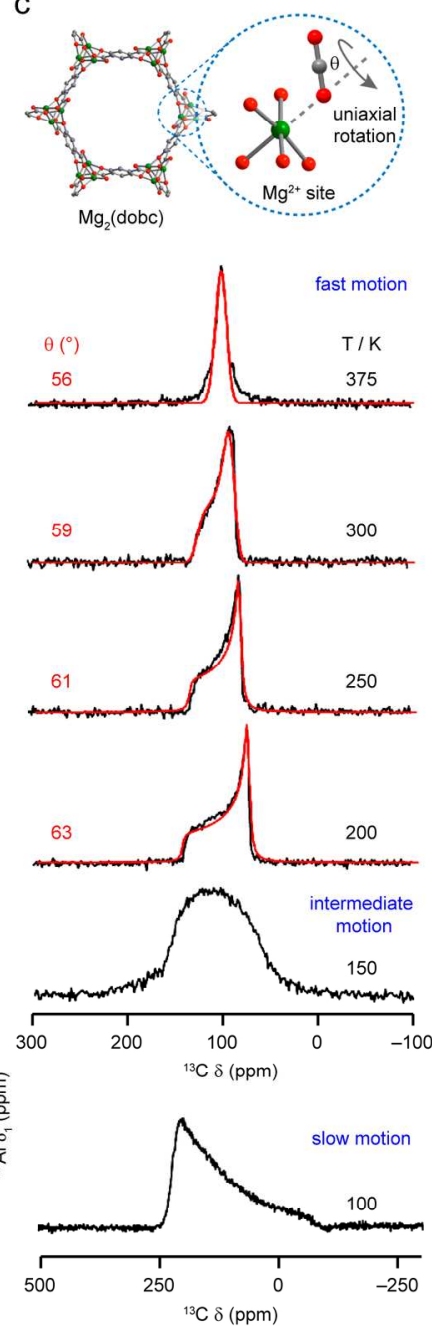

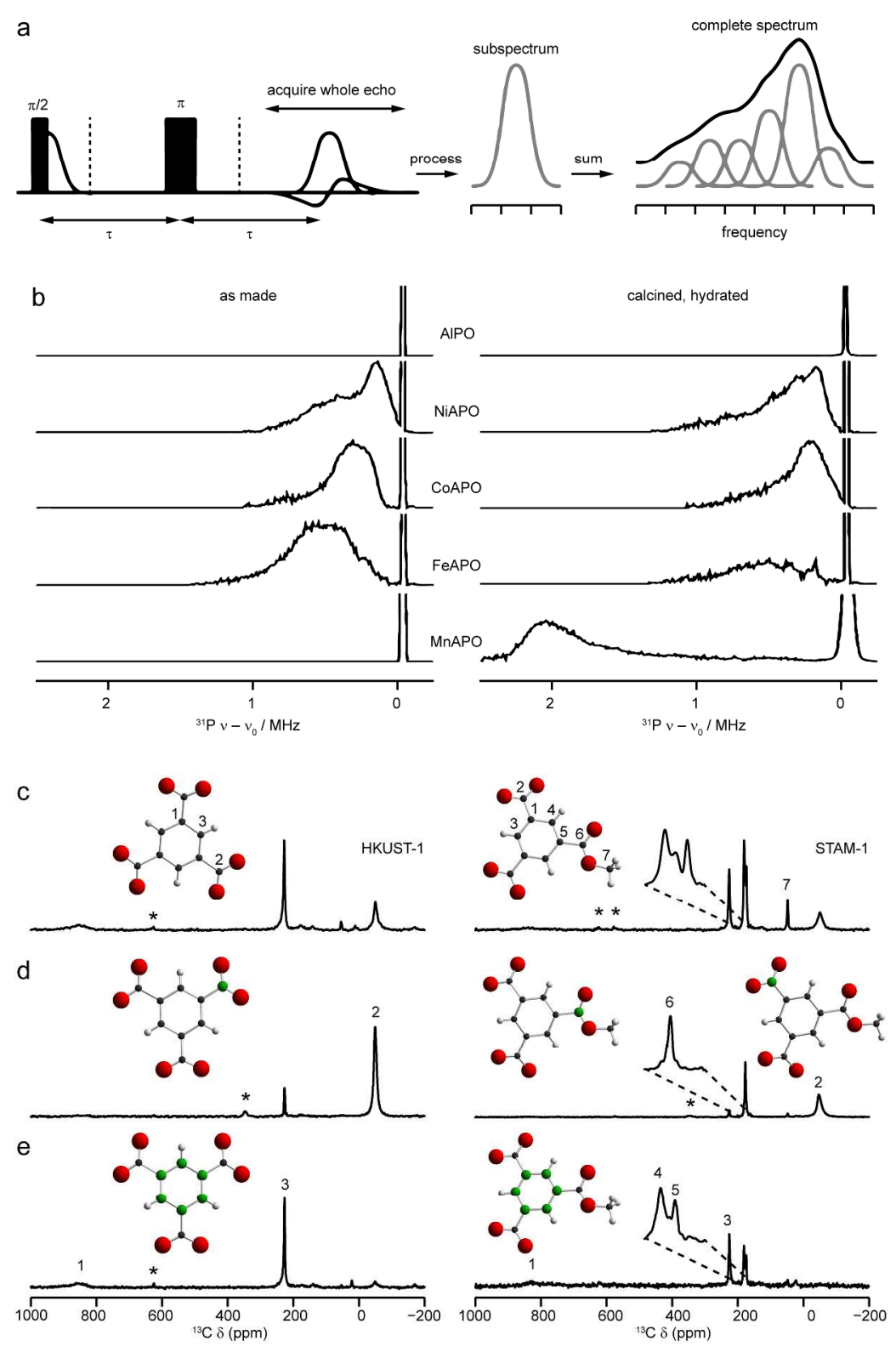

$219 \times 333 \mathrm{~mm}(300 \times 300 \mathrm{DPI})$ 


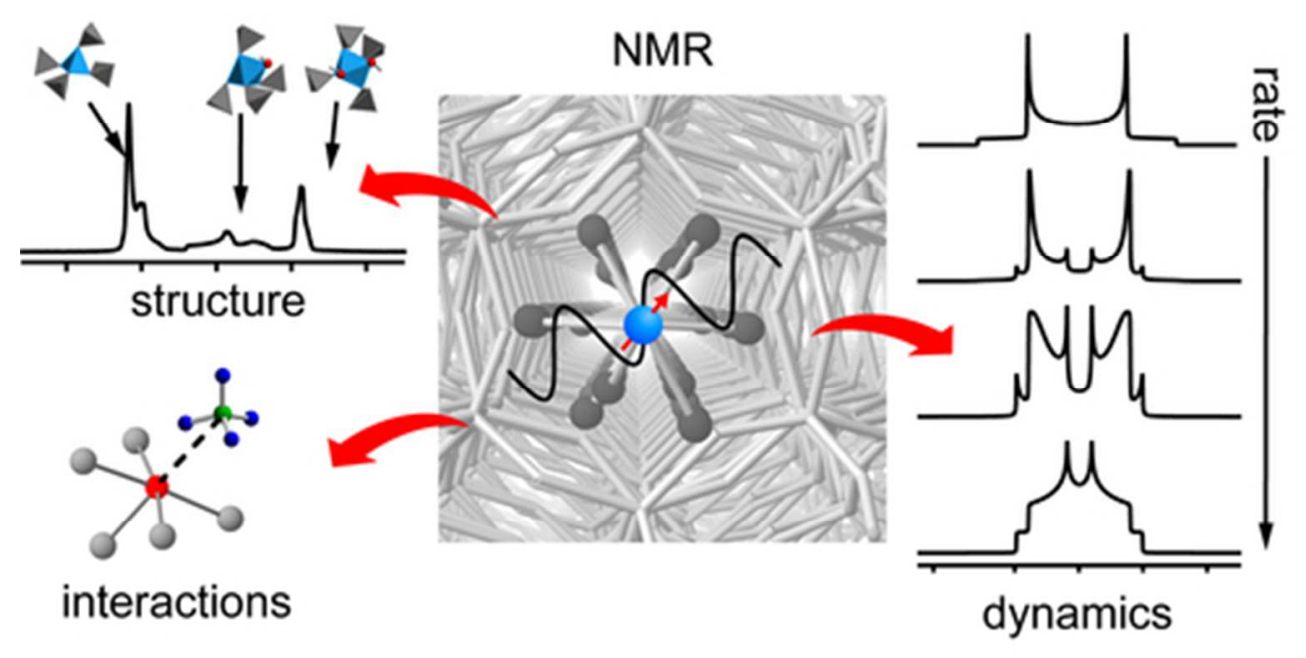

$41 \times 21 \mathrm{~mm}(300 \times 300 \mathrm{DPI})$ 